\title{
Cancer-associated mesothelial cells promote ovarian cancer chemoresistance through paracrine osteopontin signaling
}

\author{
Jin Qian, ${ }^{1}$ Bauer L. LeSavage, ${ }^{2}$ Kelsea M. Hubka, ${ }^{3}$ Chenkai Ma, ${ }^{4}$ Suchitra Natarajan, ${ }^{1}$ Joshua T. Eggold, ${ }^{1}$ Yiren Xiao, ${ }^{1}$ \\ Katherine C. Fuh, ${ }^{5}$ Venkatesh Krishnan, ${ }^{6}$ Annika Enejder, ${ }^{3}$ Sarah C. Heilshorn, ${ }^{3}$ Oliver Dorigo, ${ }^{6}$ and Erinn B. Rankin ${ }^{1,6}$ \\ ${ }^{1}$ Department of Radiation Oncology, ${ }^{2}$ Department of Bioengineering, and ${ }^{3}$ Department of Materials Science and Engineering, Stanford University, Stanford, California, USA. ${ }^{4}$ Molecular Diagnostics Solutions, \\ CSIRO Health and Biosecurity, North Ryde, New South Wales, Australia. ${ }^{5}$ Department of Obstetrics and Gynecology, Division of Cynecologic Oncology, Washington University, St. Louis, Missouri, USA. \\ ${ }^{6}$ Department of Obstetrics and Gynecology, Stanford University, Stanford, California, USA.
}

Ovarian cancer is the leading cause of gynecological malignancy-related deaths, due to its widespread intraperitoneal metastases and acquired chemoresistance. Mesothelial cells are an important cellular component of the ovarian cancer microenvironment that promote metastasis. However, their role in chemoresistance is unclear. Here, we investigated whether cancer-associated mesothelial cells promote ovarian cancer chemoresistance and stemness in vitro and in vivo. We found that osteopontin is a key secreted factor that drives mesothelial-mediated ovarian cancer chemoresistance and stemness. Osteopontin is a secreted glycoprotein that is clinically associated with poor prognosis and chemoresistance in ovarian cancer. Mechanistically, ovarian cancer cells induced osteopontin expression and secretion by mesothelial cells through TCF- $\beta$ signaling. Osteopontin facilitated ovarian cancer cell chemoresistance via the activation of the CD44 receptor, PI3K/AKT signaling, and ABC drug efflux transporter activity. Importantly, therapeutic inhibition of osteopontin markedly improved the efficacy of cisplatin in both human and mouse ovarian tumor xenografts. Collectively, our results highlight mesothelial cells as a key driver of ovarian cancer chemoresistance and suggest that therapeutic targeting of osteopontin may be an effective strategy for enhancing platinum sensitivity in ovarian cancer.

\section{Introduction}

Epithelial ovarian cancer is the leading cause of gynecological malignancy-related deaths (1). High-grade serous ovarian cancer (HGSOC) is the most prevalent form, which accounts for $70 \%$ to $80 \%$ of all mortalities (2). HGSOC usually presents at an advanced stage with malignant ascites and widespread metastases throughout the peritoneal cavity (3). The standard treatments for HGSOC are cytoreductive surgery followed by platinum and taxane-based chemotherapy (4). Although most HGSOCs are initially sensitive to first-line chemotherapy, acquired resistance to chemotherapeutic agents almost inevitably develops within 5 years (3). Thus, resistance to platinum-based chemotherapy is a major challenge in the clinical management of epithelial ovarian cancer.

The peritoneal cavity and internal organs are lined by a monolayer of cobblestone-like mesothelial cells (5). Mesothelial cells also constitute a substantial proportion of cells within the ascites fluid of HGSOC (6). Studies have suggested that mesothelial cells function as a "double-edged sword" in ovarian cancer progression. On one

Conflict of interest: OD reports receiving speakers bureau honoraria from Tesaro and AstraZeneca and is a consultant/advisory board member for Merck, Tesaro/CSK, PACT, Clovis, and Genentech.

Copyright: (C) 2021, American Society for Clinical Investigation.

Submitted: November 19, 2020 ; Accepted: June 25, 2021; Published: August 16, 2021.

Reference information: J Clin Invest. 2021;131(16):e146186.

https://doi.org/10.1172/JCl146186. hand, as the outmost layer of peritoneum, they function as a mechanical barrier preventing attachment and invasion of cancer cells into the submesothelial extracellular matrix (ECM) (5). To gain access to the submesothelial layer, disseminated ovarian cancer spheroids use myosin-generated forces to drive mesothelial cell clearance $(5,7)$. On the other hand, cancer cell-mediated reprogramming activates mesothelial cells to express factors that promote ovarian cancer proliferation, adhesion, migration, and invasion (8-10). Thus, mesothelial cells are an important cellular component of the ovarian cancer microenvironment that facilitates tumor progression.

Osteopontin (OPN, also known as SPP1) is a multifunctional matricellular phosphoglycoprotein that is overexpressed in ovarian cancer. Clinically, OPN is a serum biomarker for ovarian cancer detection (11-13). In addition, elevated OPN in blood and ascites fluid is associated with platinum resistance and poor survival in ovarian cancer (14-16). OPN signals through CD44 and integrin receptors to activate multiple downstream signaling pathways, including PI3K/AKT, MEK/ERK, and JAK/STAT pathways (17-20). Functionally, OPN promotes metastasis, proliferation, stem-like phenotypes, chemoresistance, radiation resistance, and immune suppression in a variety of cancer types $(21,22)$. However, the regulation and functional role of OPN in ovarian cancer are poorly defined. Here, we report that bidirectional signaling between ovarian cancer cells and mesothelial cells promotes ovarian cancer chemoresistance and stemness through OPN. Our findings reveal that mesothelial cells are an abundant source of 


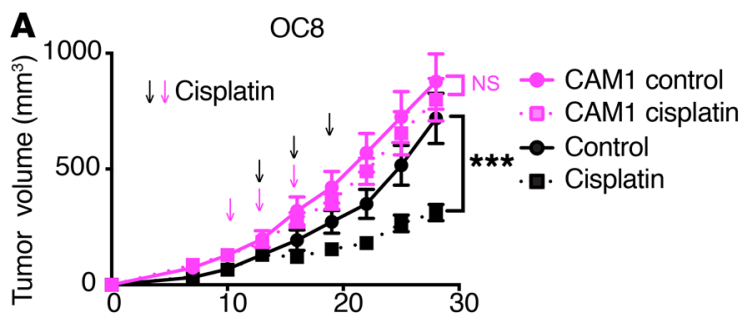

Days after cell inoculation
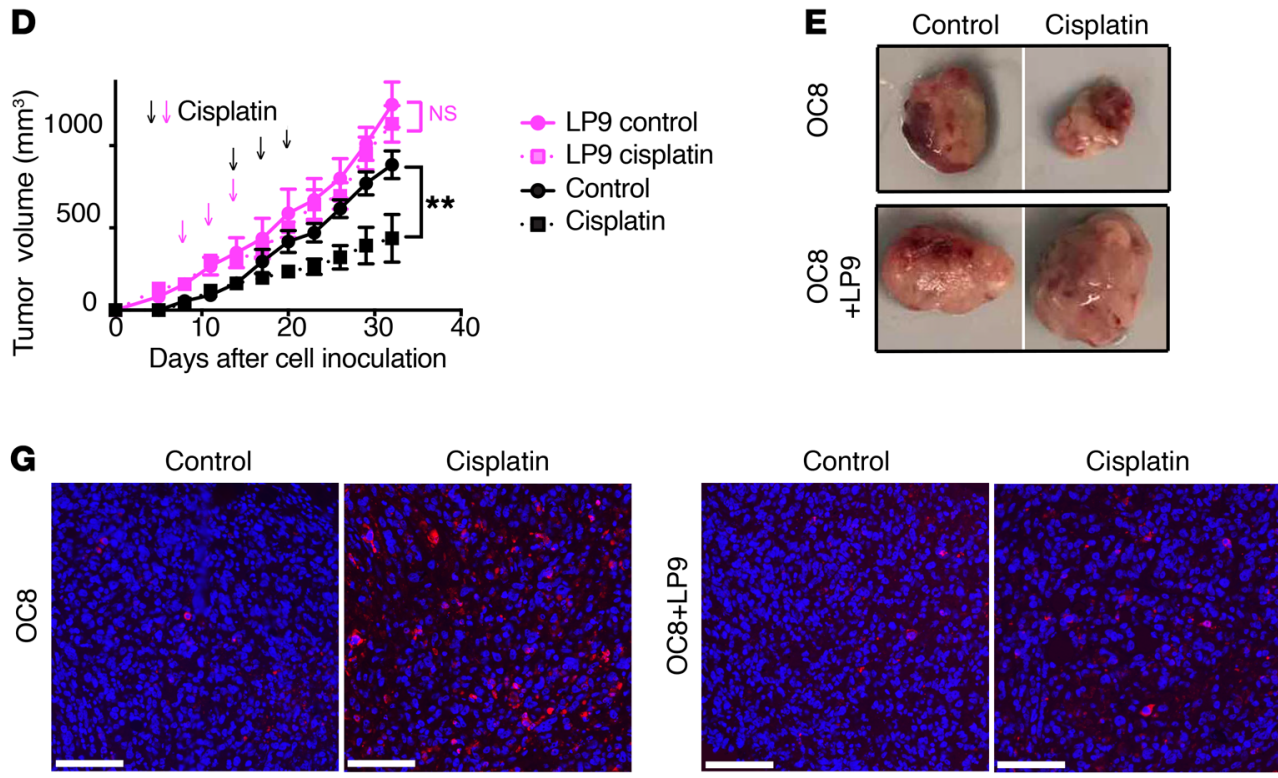

$\rightarrow$ Control

- Cisplatin
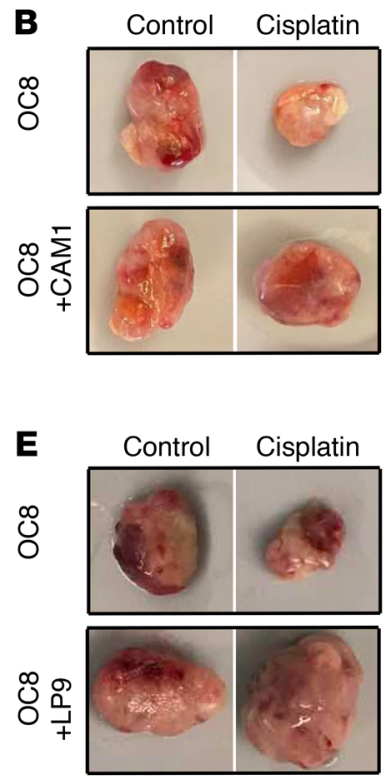

E

I
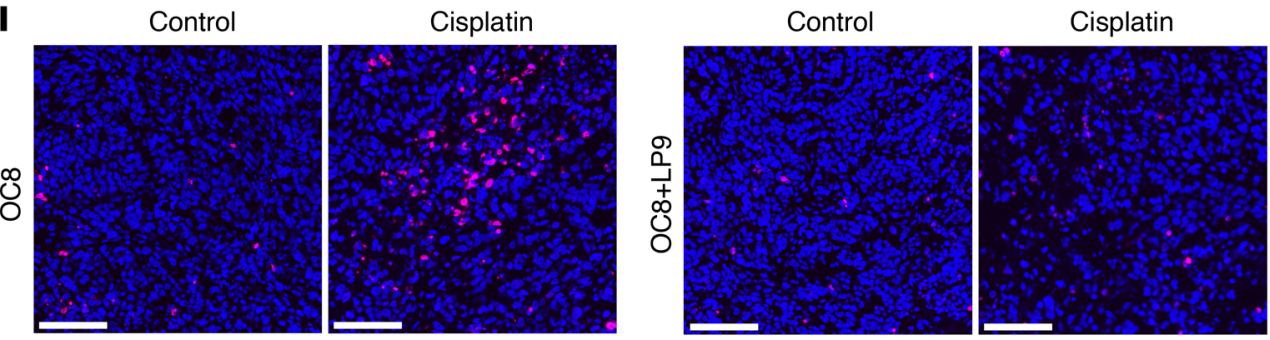
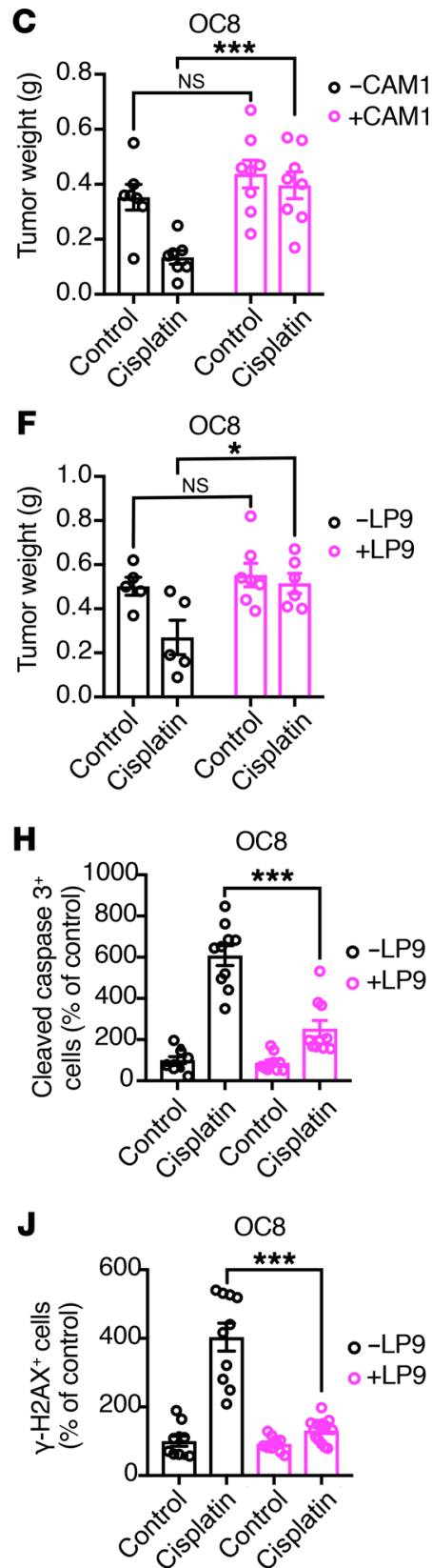

Figure 1. Cancer-associated mesothelial cells promote ovarian cancer platinum resistance. (A-F) Effect of primary CAM1 (A-C) or LP9 (D-F) coinjection on cisplatin response of primary OC8 HCSOC cells in vivo. OC8 cells or OC8 cells plus LP9 or CAM1 mesothelial cells were injected subcutaneously into female immunodeficient mice and treated with or without cisplatin every 3 days for 3 cycles. Tumor growth curves are shown in $\mathbf{A}(n=7-8$ mice per group) and $\mathbf{D}$ ( $n=5-7$ mice per group). Representative xenograft images are shown in $\mathbf{B}$ and $\mathbf{E}$. Xenograft weights at the end point are shown in $\mathbf{C}$ and $\mathbf{F}$. Arrows show scheme of cisplatin treatment: magenta arrows for mesothelial cell-coinjected groups, black arrows for OC8 cell alone groups. (G-J) Representative images and quantification of cleaved caspase-3 ( $\mathbf{G}$ and $\mathbf{H}$ ) and $\gamma-\mathrm{H} 2 \mathrm{AX}$ ( $\mathbf{I}$ and $\mathbf{J})$ immunofluorescence staining in OC8 and LP9 coinjected tumors. Scale bars: $100 \mu \mathrm{m}$. Quantification of positive cells (percentage of control) is based on 10 random fields from more than 3 tumors in each group. Each dot represents 1 field. Nuclei were stained with DAPI (blue). Data are presented as mean \pm SEM. ${ }^{*} P<0.05 ;{ }^{* *} P<0.01$; ${ }^{* *} P<0.001,2$-way ANOVA (A, C, D, and F) and 2-tailed Student's $t$ test (H and J).

OPN in the ovarian cancer microenvironment and that therapeutic targeting of OPN may be an effective strategy for enhancing platinum sensitivity in ovarian cancer.

\section{Results}

Cancer-associated mesothelial cells promote ovarian cancer chemoresistance. To investigate the contribution of mesothelial cells to platinum resistance, we isolated cancer-associated mesothelial cells (CAM1 and CAM2) directly from the malignant ascites of ovarian cancer patients (Supplemental Table 1; supplemental material available online with this article; https://doi.org/10.1172/JCI146186DS1). Immunostaining confirmed that the primary mesothelial cells were positive for the mesothelial markers calretinin $(8,23,24)$, cytokeratin, and vimentin (25) and were negative for markers of other cell types, including immune, endothelial, and fibroblast cells (Supplemental Figure 1A). Primary mesothelial cells displayed a char- 
A

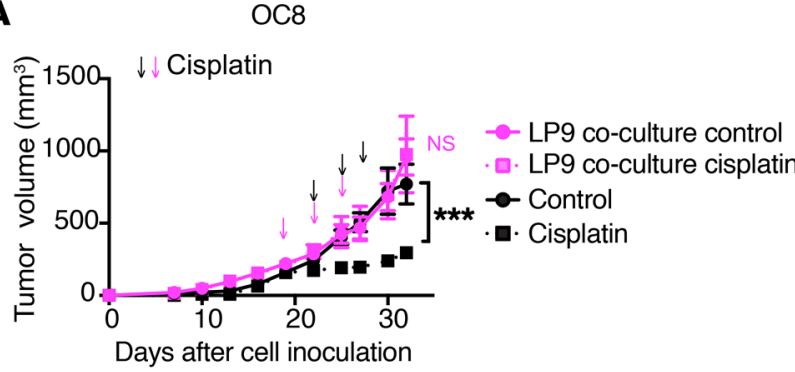

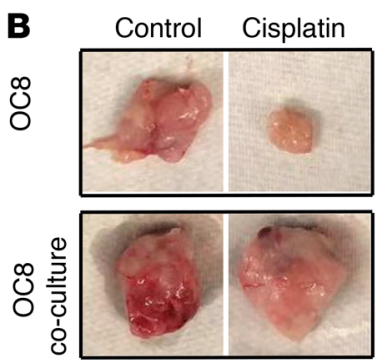
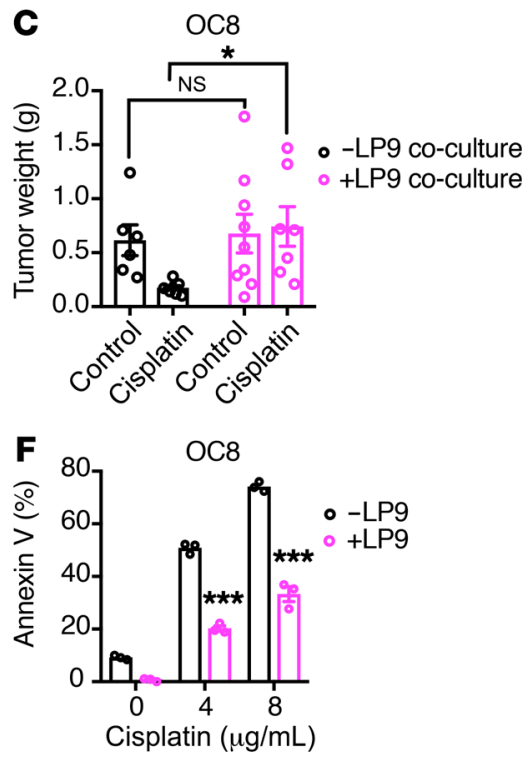

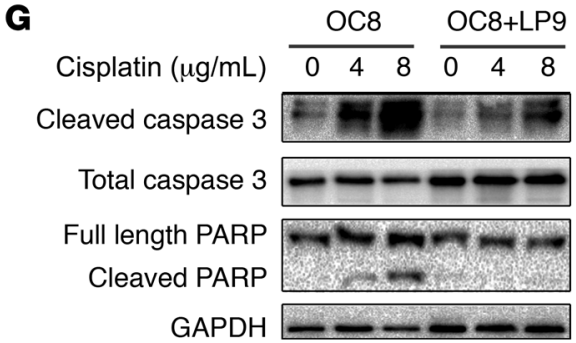

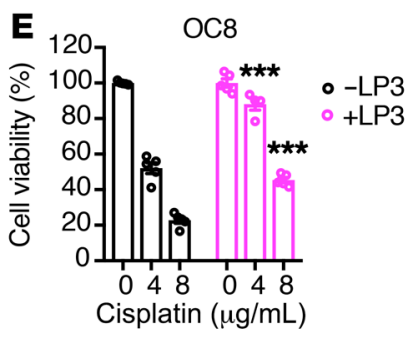

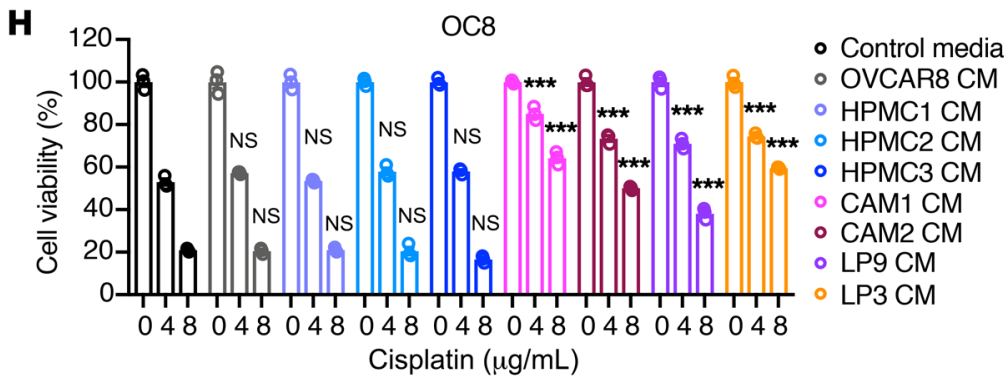

Figure 2. Secreted factor or factors from cancer-associated mesothelial cells promote ovarian cancer chemoresistance. (A-C) Effect of LPS coculture on cisplatin resistance of OC8 cells in vivo. Tumor cells were indirectly cocultured with or without LP9 mesothelial cells in vitro and then were injected subcutaneously into immunodeficient mice, followed by treatment with or without cisplatin every 3 days for 3 cycles. Tumor growth curve is shown in $\mathbf{A}(n=6-9$ mice per group). Representative xenograft images are shown in B. Xenograft weights at the end point are shown in $\mathbf{C}$. Arrows show scheme of cisplatin treatment: magenta arrows for mesothelial cell-conditioned groups, black arrows for unconditioned OC8 cell groups. (D and E) Effect of LP9 or LP3 coculture on the cisplatin sensitivity of OC8 cells. Cell viability is normalized to its untreated control and statistically compared with OC8 monoculture group ( $n$ = 3-5). (F) Percentages of annexin $\mathrm{V}^{+}$apoptotic OC8 cells with or without LP9 preconditioning. Each group is statistically compared with OC8 monoculture group $(n=3)$. (C) Western blot analysis of cisplatin-induced apoptotic markers in OC8 after LP9 coculture. (H) Effect of conditioned media (CM) from HPMCs and cancer-associated mesothelial cells on OC8 cisplatin sensitivity. Cell viability is normalized to its untreated control and statistically compared with control media group $(n=3)$. Data are presented as mean \pm SEM. ${ }^{* *} P<0.05 ;{ }^{* * *} P<0.001,2$-way ANOVA $(\mathbf{A}, \mathbf{C}-\mathbf{F}$, and $\mathbf{H})$.

acteristic cobblestone-like morphology in culture without EGF stimulation, further supporting their mesothelial cell identity (Supplemental Figure 1B and ref. 25). In addition, we obtained the previously characterized LP9 and LP3 mesothelial cells derived from ovarian cancer patient ascites fluid for our studies (26).

We first examined the impact of cancer-associated mesothelial cells on ovarian cancer cell sensitivity to cisplatin in tumor xenograft studies. Female mice were subcutaneously injected with the previously characterized primary HGSOC cells (OC8) alone or together with CAM1 or LP9 mesothelial cells (27). We confirmed that CAM1 and LP9 mesothelial cells were not tumorigenic when injected alone into female $\mathrm{Rag}^{--} \mathrm{IL} 2 \mathrm{rg}^{-/}$immunodeficient mice (data not shown, ref. 26). When tumors reached 100 to $200 \mathrm{~mm}^{3}$, the mice were treated with cisplatin or vehicle. While cisplatin treatment inhibited the growth of $\mathrm{OC} 8$ tumors, cisplatin treatment did not affect the growth of OC8-CAM1 or OC8-LP9 coinjected tumors (Figure 1, A-F). Coinjection of OC8 cells with LP9 mesothelial cells reduced cisplatin-induced apoptosis and
DNA damage (Figure 1, G-J). Similarly to what occurred in our findings above, coinjection of OVCAR8 ovarian cancer cells with LP9 mesothelial cells reduced the efficacy of cisplatin treatment in vivo (Supplemental Figure 1, C-E). It should be noted that the origin of the OVCAR8 ovarian cancer cell line is not clear (28). While OVCAR 8 cells are $\mathrm{p} 53$ deficient and have been utilized as an HGSOC cancer cell line, OVCAR8 cells also have mutations that are associated with nonserous ovarian cancer (29-31). Together, these findings demonstrate that cancer-associated mesothelial cells promote ovarian cancer cisplatin resistance in OC8 and OVCAR8 xenograft models.

Cancer-associated mesothelial cells promote ovarian cancer cell chemoresistance through secreted factor(s). To determine whether the chemoprotective effect of cancer-associated mesothelial cells is mediated by secreted factors, we compared the platinum sensitivity of ovarian cancer cells cultured alone or in indirect coculture with that of cancer-associated mesothelial cells (Supplemental Figure 2A). OC8 cells cocultured with LP9 mesothelial cells prior to tumor 
A

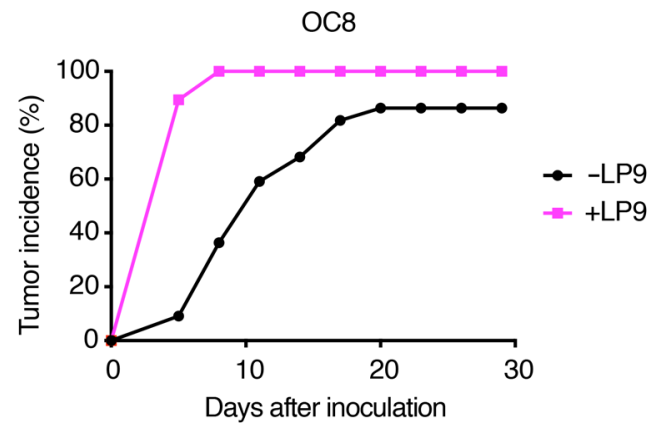

C

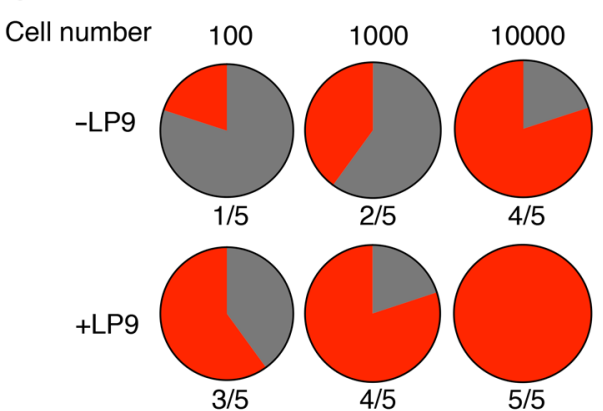

E
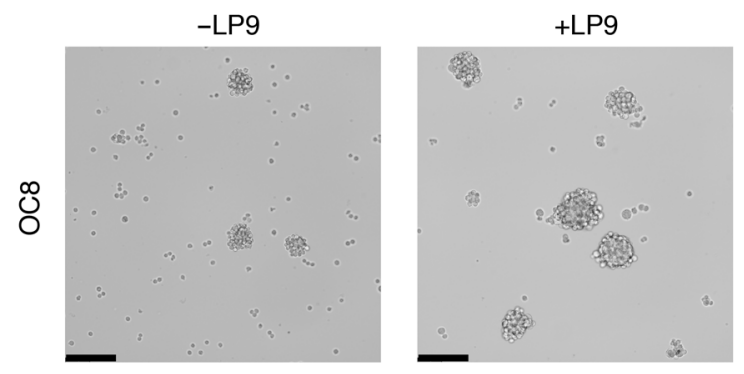

$\mathbf{F}$
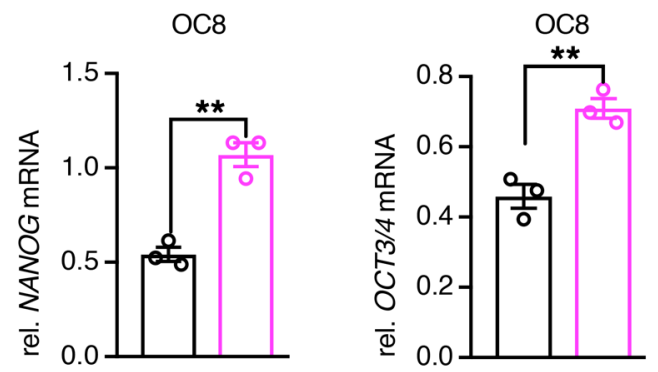

B

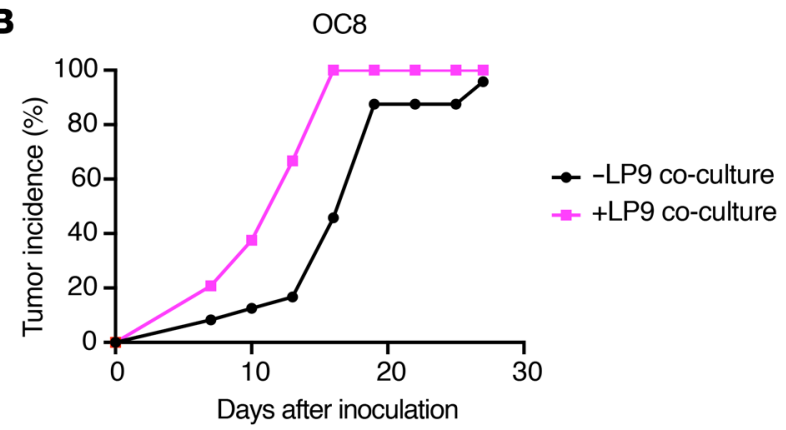

D
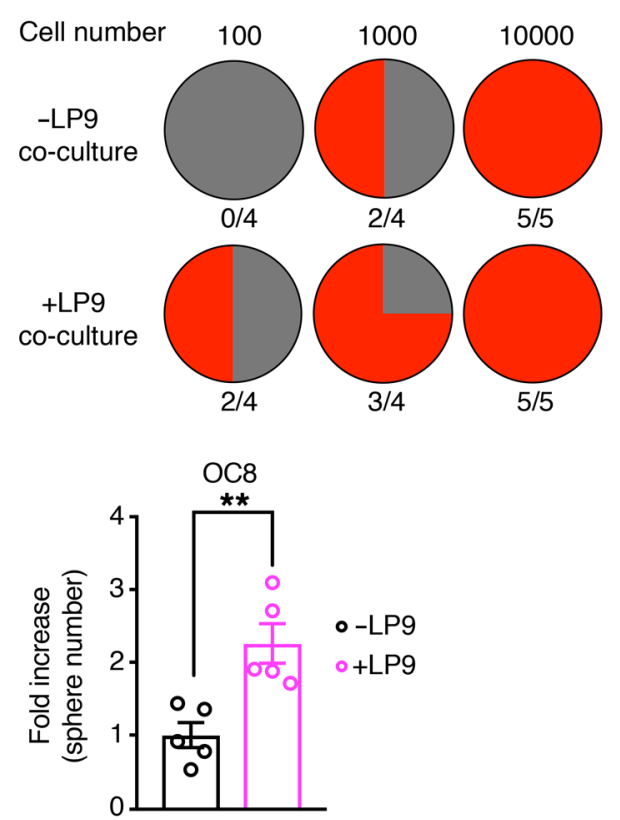

Figure 3. Cancer-associated mesothelial cells promote ovarian cancer stemness through secreted factors. (A and B) Effect of $L P 9$ coinjection (A, $n=20-22$ mice per group) or coculture (B, $n=24$ mice per group) on OC8 tumor incidence in immunodeficient mice. (C and $\mathbf{D})$ In vivo limiting dilution assays showing tumor formation rate (red portion) of LP9 coinjected (C) or in vitro cocultured (D) OC8 tumors at indicated cancer cell numbers ( $n=4-5$ mice per group). (E) Sphere-formation assay of OC8 cells after LP9 coculture. Representative sphere images and quantification of sphere number fold increase $(n=5)$. Scale bars: $200 \mu \mathrm{m}$. (F) Real-time PCR analysis showing relative mRNA expression of the stemness markers NANOG, OCT3/4, SOX2, and ALDH1A1 in OC8 after LP9 coculture, as normalized to GAPDH mRNA $(n=3)$. Data are presented as mean $\pm \mathrm{SEM} .{ }^{*} P<0.05 ;{ }^{* *} P<0.01 ;{ }^{* *} P<0.001,2$-tailed Student's $t$ test $(\mathbf{E}$ and $\mathbf{F})$.

injection were resistant to cisplatin treatment compared with OC8 cells grown as single cultures (Figure 2, A-C). In addition, cancer-associated mesothelial cell (LP9, LP3, or CAM2) coculture reduced the cisplatin sensitivity of OC8 and OVCAR8 cells in vitro (Figure 2, D and E, and Supplemental Figure 2, B-D). LP9 coculture reduced cisplatin-induced cell apoptosis in OC8 and OVCAR8 cancer cells in vitro (Figure 2, F and G, and Supplemental Figure 2, E and F).
As most ovarian cancer patients eventually succumb to resistance to multiple chemoreagents (3), we extended our studies to include additional chemotherapeutic agents utilized in the clinical management of ovarian cancer. We observed similar protective effects of LP9 and LP3 mesothelial cells on ovarian cancer cell sensitivity to carboplatin in both OC8 and OVCAR8 cells and to paclitaxel in OVCAR8 cells (Supplemental Figure 3, A-D). 
A

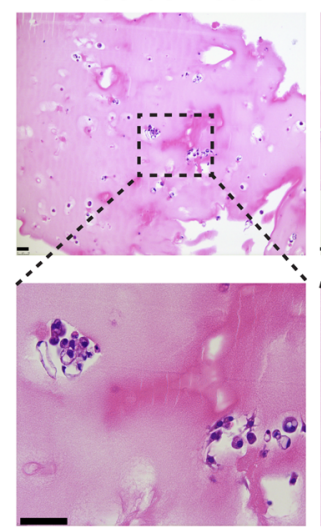

D
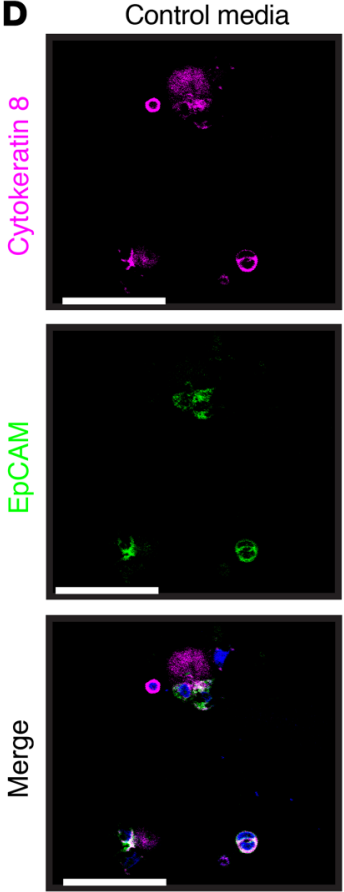

CAM1 CM

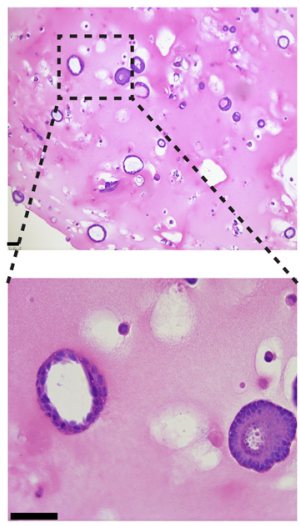

CAM1 CM
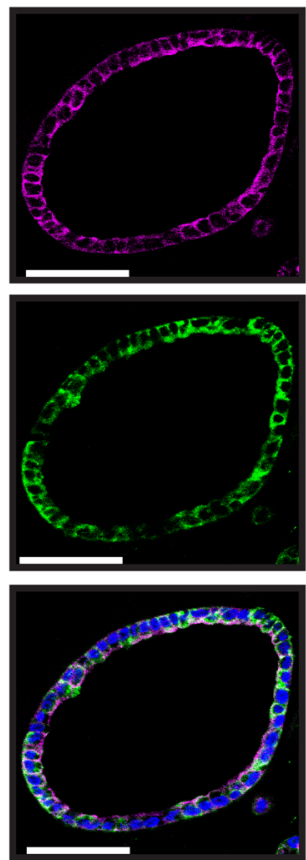

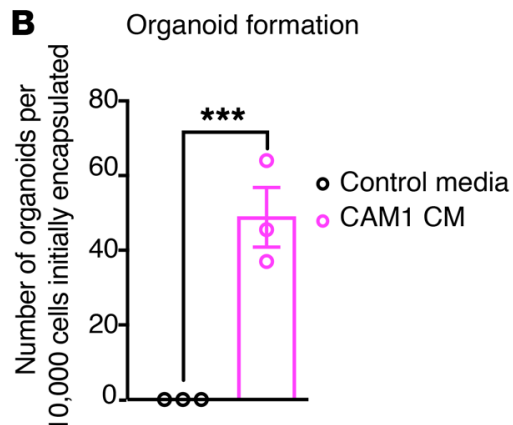

C Organoid diameter

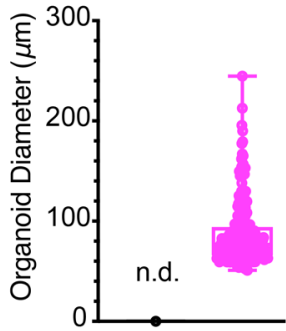

- Control media

- CAM1 CM
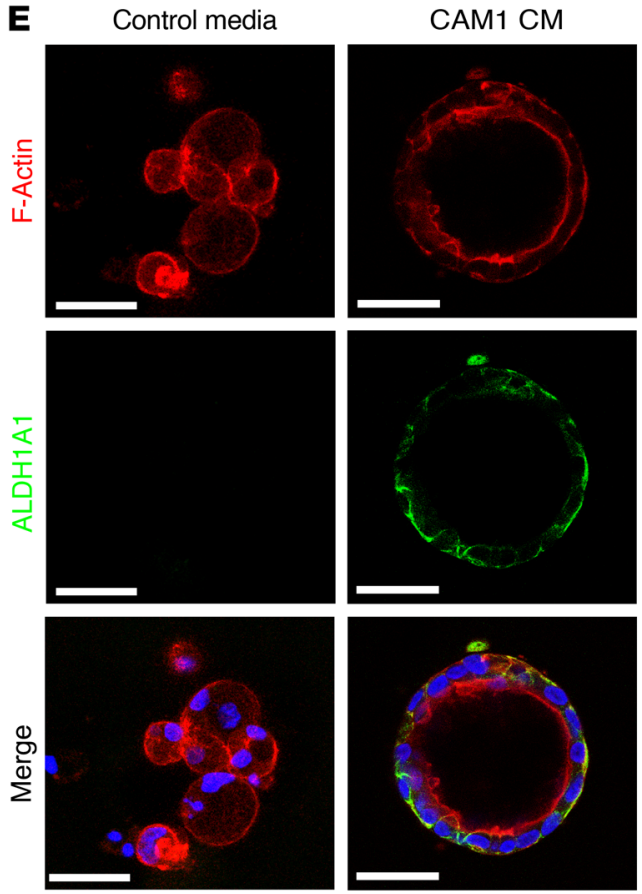

Figure 4. Cancer-associated mesothelial cells promote ovarian cancer organoid formation through secreted factors. (A) Representative H\&E images of ovarian cancer organoids in 3D. Scale bars: $100 \mu \mathrm{m}$ (upper panels); $50 \mu \mathrm{m}$ (lower panels). (B) Ovarian cancer organoid formation per 10,000 initially encapsulated EpCAM+ ovarian cancer cells with addition of paired CAM1-conditioned media or control media ( $n=3$ ). (C) Ovarian cancer organoid diameter (defined as cell clusters $>50 \mu \mathrm{m}$ in diameter with lumen) with addition of paired CAM1-conditioned media or control media. Each point represents 1 organoid. n.d., none detected. (D) Immunofluorescent staining of cytokeratin-8 (magenta) and EpCAM (green) in ovarian cancer organoids grown in CAM1-conditioned media or in control media. Nuclei were stained with DAPI (blue). Scale bars: 50 um. (E) Immunofluorescence of F-actin (red) and stemness marker ALDH1A1 (green) in ovarian cancer organoids grown in CAM-conditioned media or in control media. Nuclei were stained with DAPI (blue). Scale bars: $50 \mu$ m. Data are presented as mean \pm SEM. ${ }^{* *} P<0.001,2$-tailed Student's $t$ test (B).

Next we sought to determine whether human primary mesothelial cells (HPMCs) derived from patients with benign disease have a similar protective effect on ovarian cancer cell platinum sensitivity (8). Compared with the conditioned media collected from cancer-associated mesothelial cells (CAM1, CAM2, LP9, and LP3), the conditioned media from 3 independent HPMCs did not affect ovarian cancer cell (OC8, OVCAR8, CAOV3, or SNU119) cisplatin sensitivity in vitro (Figure $2 \mathrm{H}$ and Supplemental Figure 4, A-C). Overall, our results demonstrate that cancer-associated mesothelial cells promote ovarian cancer chemoresistance through soluble factor(s).

Cancer-associated mesothelial cells promote ovarian cancer stemness through secreted factor(s). Multidrug resistance has been linked to cancer stemness (32). To determine whether cancer-associated mesothelial cells induce ovarian cancer stemness, we compared the tumor incidence of ovarian cancer cells injected alone or together with cancer-associated mesothelial cells. LP9 and CAM1 mesothelial cells enhanced the tumor-formation rate of OC8 and OVCAR8 xenografts (Figure 3A and Supplemental Figure 5, A and B). In addition, preconditioning OC8 cells with LP9 cells in vitro also enhanced OC8 tumor formation (Figure 3B). A gold standard assay to assess cancer stem cell (CSC) enrichment is the in vivo limiting dilution assay, which examines tumorigenic rate with serial dilutions of cancer cells (33). In this assay, the tumor formation rate was higher at low cell numbers in OC8 cells that were 
A

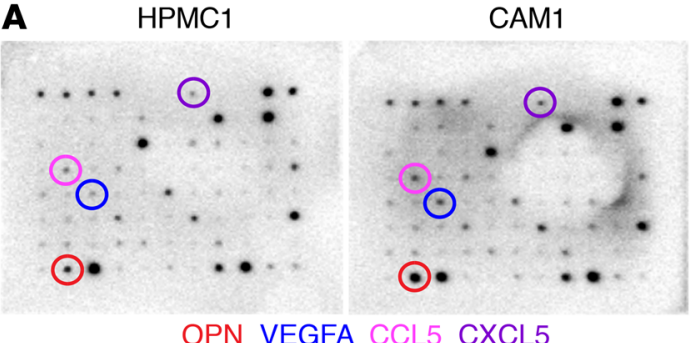

OPN VEGFA CCL5 CXCL5

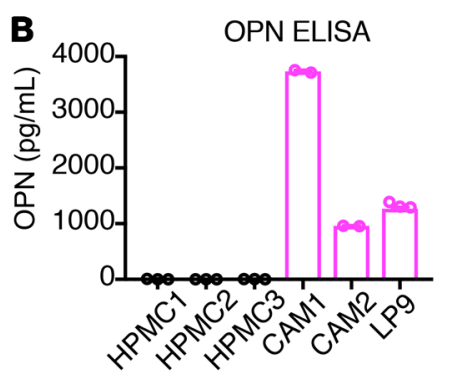

OPN mRNA

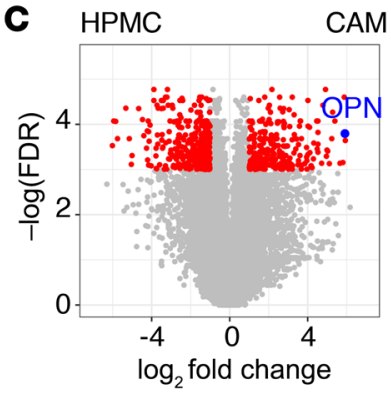

D

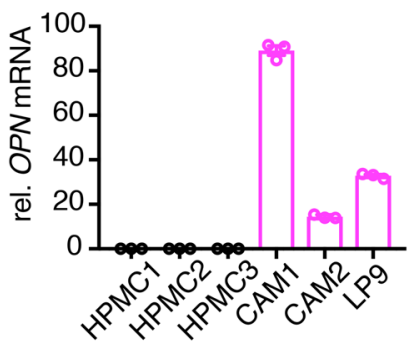

E
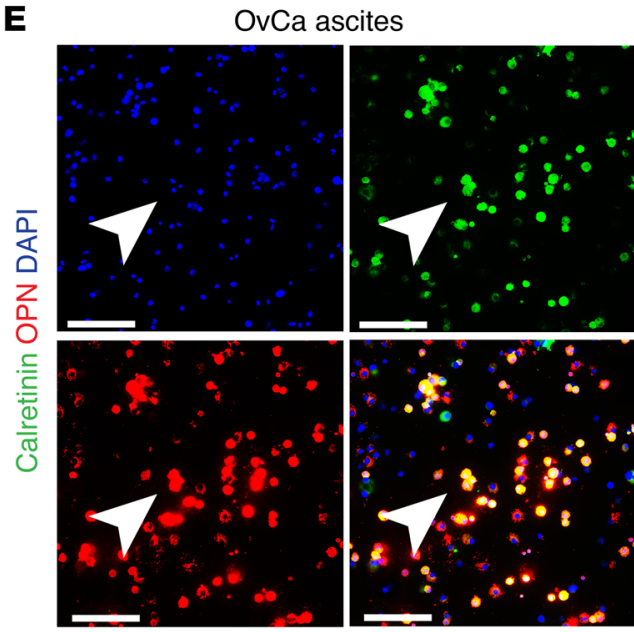

$\mathbf{F}$
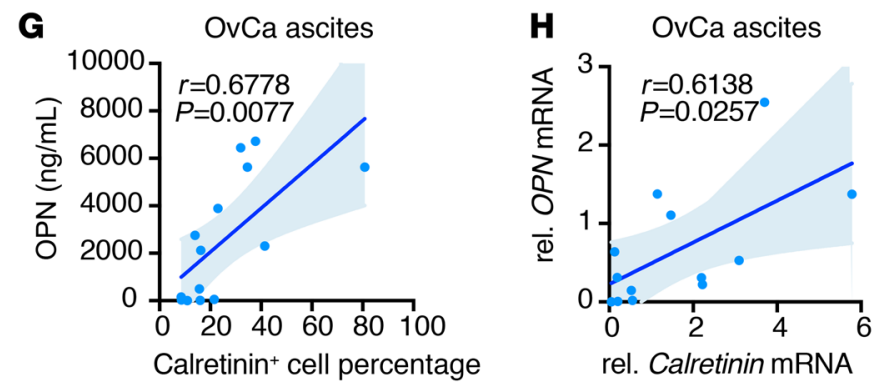

Figure 5. Cancer-associated mesothelial cells secrete OPN. (A) Cytokine array in conditioned media of HPMC1 and CAM1. Circles highlight cytokine of the highest increase in CAM1compared with HPMC1-conditioned media in each individual color. (B) Quantification of OPN concentration in conditioned media from HPMCs and CAMs by ELISA ( $n=2$ or 3 ). (C) Volcano plot of RNA-Seq showing differential gene expression in CAMs versus HPMCs (GSE84829). (D) Real-time PCR analysis of relative mRNA expression of OPN in HPMCs and CAMs, as normalized to GAPDH mRNA $(n=3)$. (E) Immunofluorescence of OPN (red) and mesothelial cell marker calretinin (green) in total ascites cells from HCSOC patients. Arrowheads denote costained mesothelial cells. Nuclei were stained with DAPI (blue). Scale bars: $100 \mu \mathrm{m}$. (F) Percentages of total OPN-positive cells in paired calretinin ${ }^{+}$and calretinin ${ }^{-}$ascites cells of ovarian cancer patients $(n=14)$. (C) Correlation of ascites OPN concentration and calretinin ${ }^{+}$cell percentages in total ascites cells of ovarian cancer patients ( $n=14$; Pearson's correlation). (H) Correlation of OPN and calretinin expression in total ascites cells of ovarian cancer patients ( $n=13$; Pearson's correlation). Data are presented as mean $\pm \mathrm{SEM}$. ${ }^{* *} P<0.001$, 2-tailed Student's $t$ test (F).

enhanced the expression of stemness markers, including NANOG, OCT3/4, SOX2 and, ALDH1A1 in ovarian cancer cells (OC8 and OVCAR8 cells; Figure 3F, Supplemental Figure 5, F-H, and refs. $32,34,35)$. ALDH activity is a functional marker of ovarian CSCs $(33,36)$. LP9 mesothelial cells increased the percentages of $\mathrm{ALDH}^{+} \mathrm{OC} 8$ and OVCAR8 cells in vitro (Supplemental Figure 5I) and in coinjected or preconditioned tumor xenografts (Supplemental Figure 5, J-L).

Finally, we utilized a 3D organoid approach to determine whether patient-matched cancer-associated mesothelial cells facilitate the initiation and growth of primary ovarian cancer organoids. We sorted $\mathrm{EpCAM}^{+}$cancer cells from ovarian cancer patient ascites and cultured them in 3D hydrogels

coinjected or preconditioned with LP9 cells compared with OC8 cells alone, suggesting that cancer-associated mesothelial cells promote ovarian cancer cell stemness through secreted factors (Figure 3, C and D). In vitro sphere formation correlates with the enrichment of ovarian CSCs (32). We observed that cancer-associated mesothelial cell (LP9 and LP3) indirect cocultures enhanced ovarian cancer cell (OC8 and OVCAR8) tumor-sphere formation when seeded under low-attachment conditions (Figure $3 \mathrm{E}$ and Supplemental Figure 5, C-E). At the molecular level, cancer-associated mesothelial cell (LP9, LP3, and CAM2) indirect cocultures with the stimulation of patient-matched primary mesothelial cell-conditioned media. Strikingly, the patient-derived conditioned media induced the formation of organoids with well-organized lumen structures compared with smaller, unorganized cell clusters in the samples with control media (Figure 4A). Moreover, the efficiency of ovarian cancer cell organoid formation and growth was markedly enhanced with the patient-derived conditioned media compared with control media (Figure 4, B and C). Organoids and cell clusters stained positive for EpCAM and the ovarian cancer marker cytokeratin-8, confirm- 

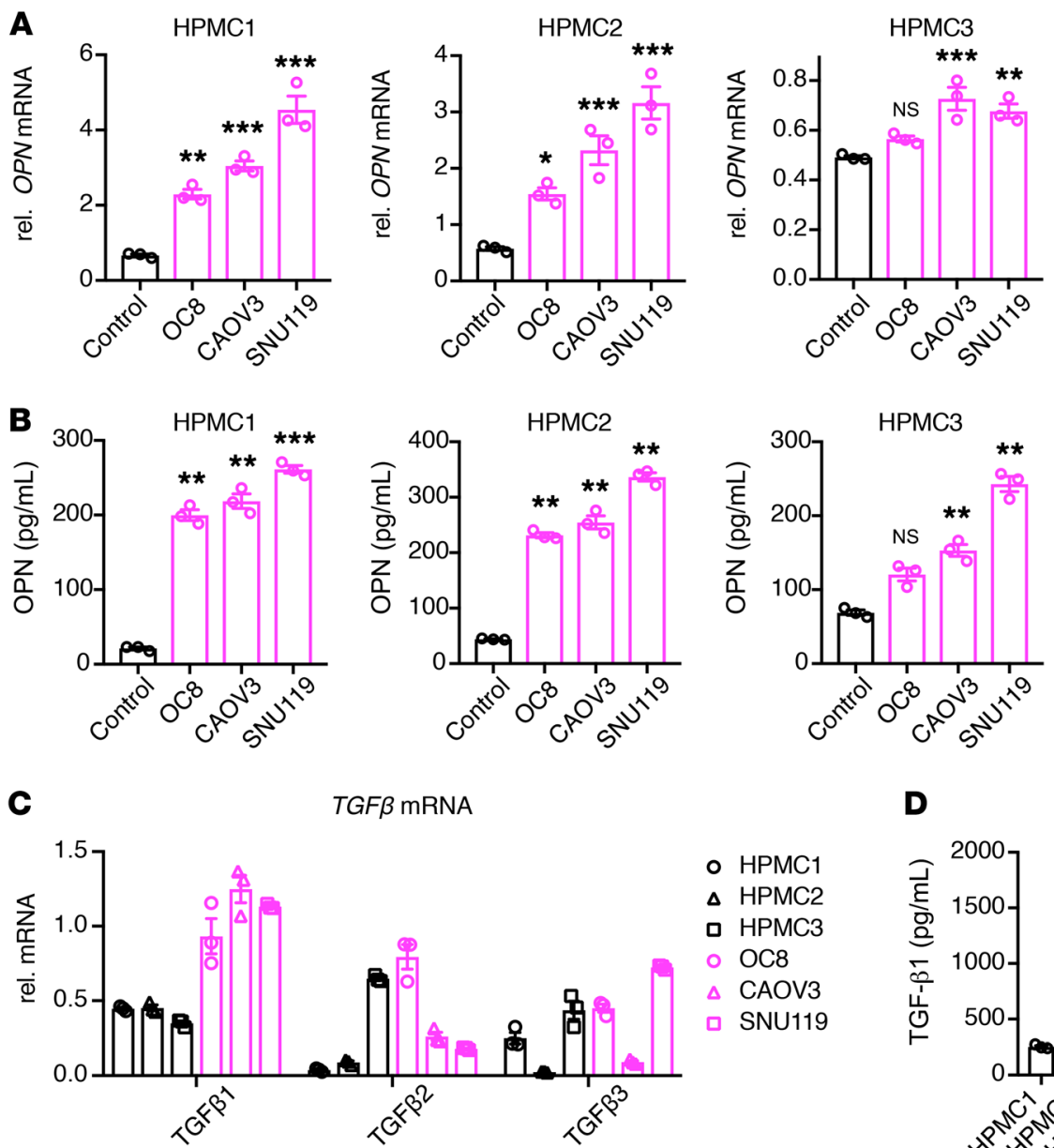

\section{D}

TGFß1 ELISA
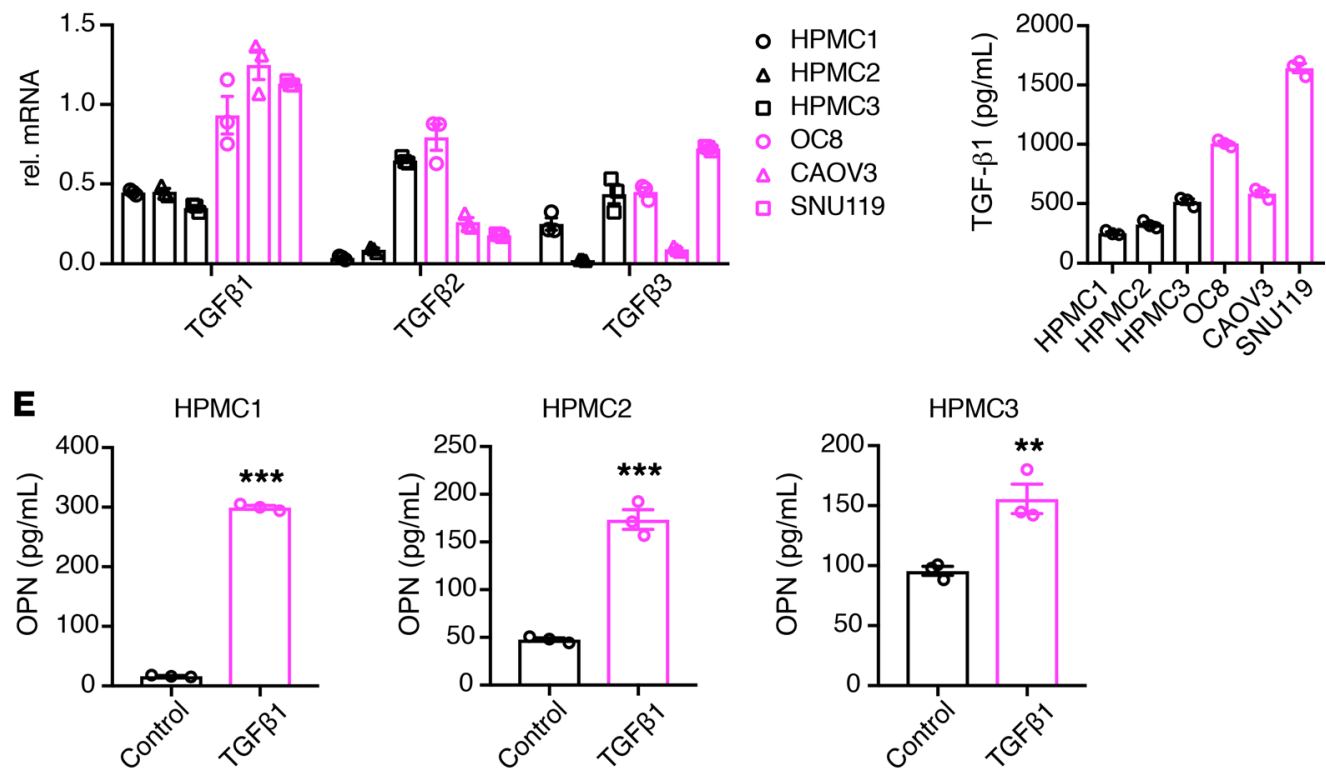

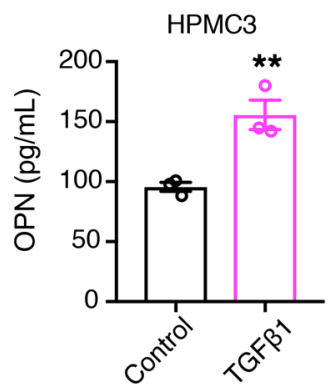

HPMC2

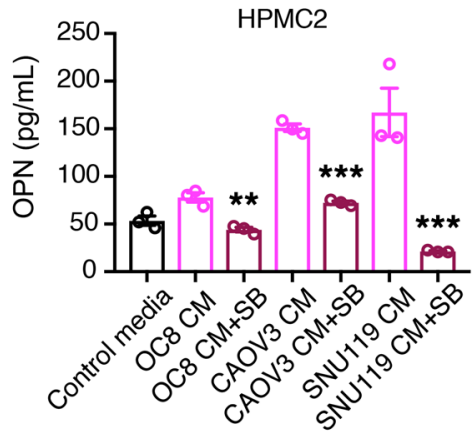

Figure 6. Ovarian cancer cells induce OPN expression in mesothelial cells through TCF- $\beta$ signaling. (A) Real-time PCR analysis of relative OPN mRNA expression in HPMCs alone or cocultured with ovarian cancer cells, as normalized to GAPDH mRNA and statistically compared with HPMC cultured-alone group $(n=3)$. (B) ELISA quantification of OPN concentration in conditioned media from HPMCs alone or HPMCs cocultured with various ovarian cancer cells. Each group is statistically compared with HPMC cultured-alone group ( $n=$ 3). (C) Real-time PCR analysis of relative TCFB1, TCFB2, and TCFB3 expression in HPMCs or ovarian cancer cells, as normalized to GAPDH mRNA $(n=3)$. (D) ELISA quantification of TCF- $\beta 1$ concentration in conditioned media from HPMCs or ovarian cancer cells ( $n$ = 3). (E) ELISA quantification of OPN concentration in conditioned media from HPMCs treated with PBS or TCF- $\beta 1(10 \mathrm{ng} / \mathrm{ml})$ for 3 days $(n=3)$. (F) ELISA quantification of OPN concentration in conditioned media from HPMCs cultured in control media, HPMCs treated with ovarian cancer cellconditioned media, or HPMCs treated with ovarian cancer cellconditioned media plus SB431542 (SB). Group with ovarian cancer cell-conditioned media plus SB431542 treatment is statistically compared with respective ovarian cancer cell-conditioned media treated-alone group. Data are presented as mean \pm SEM. ${ }^{*} P$ $<0.05$; ${ }^{*} P<0.01$; ${ }^{* *} P<0.001$, 1-way ANOVA (A, B, and $\mathbf{F}$ ) and 2-tailed Student's $t$ test (E).

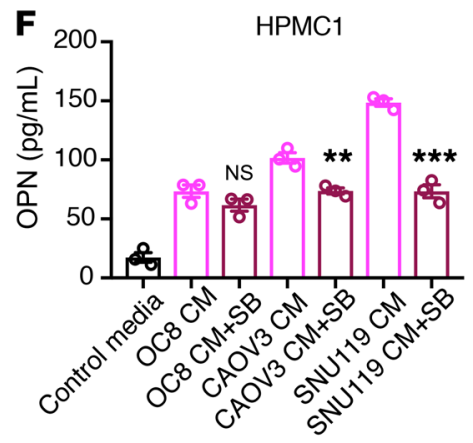

ing their ovarian cancer epithelial origin (Figure 4D). Interestingly, only the organoids treated with the patient-derived mesothelial cell-conditioned media expressed the ovarian CSC markers ALDH1A1 and CD44, suggesting the CSC population was enriched in these samples (Figure 4E, Supplemental Figure $5 \mathrm{M}$, and Supplemental Video 1). Collectively, our data suggest that cancer-associated mesothelial cell-derived factor or factors promote ovarian cancer stemness. 
A

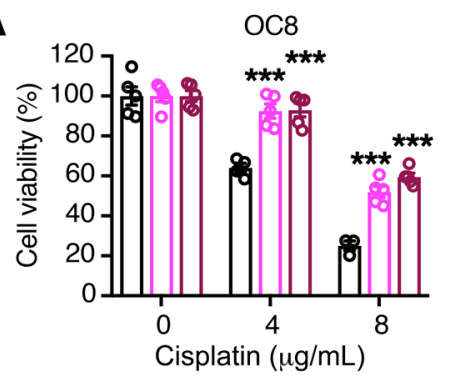

B - Control - $100 \mathrm{ng} / \mathrm{mL}$ OPN - $1000 \mathrm{ng} / \mathrm{mL}$ OPN

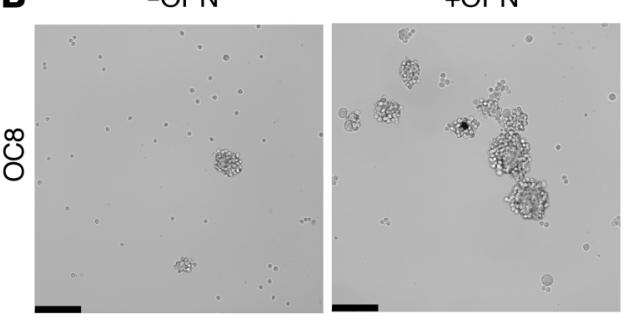

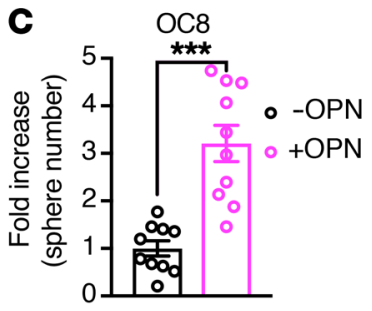

D
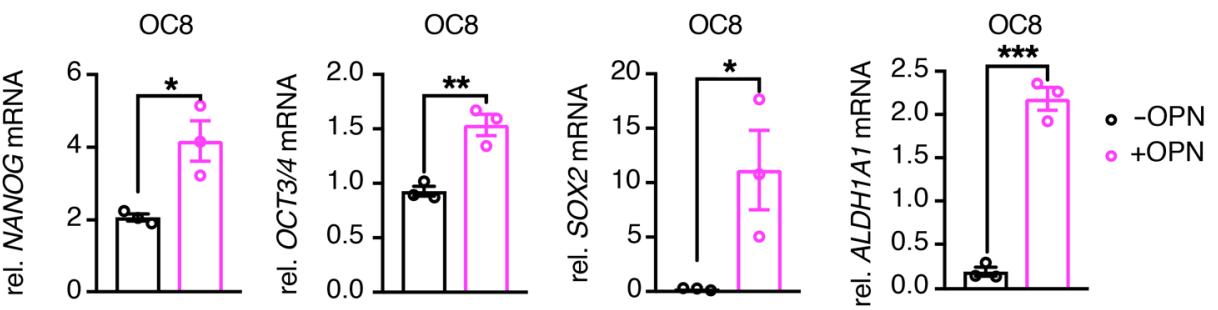

E

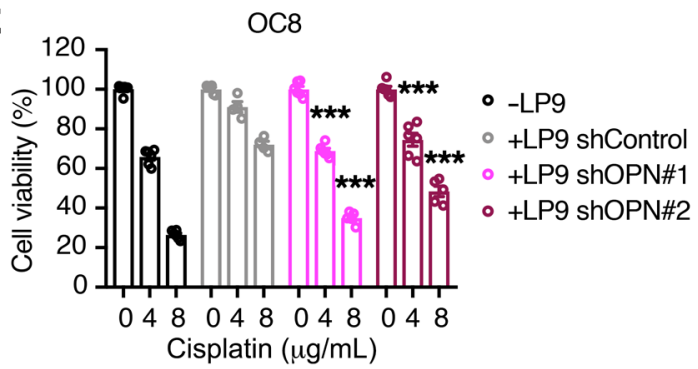

$\mathbf{F}$

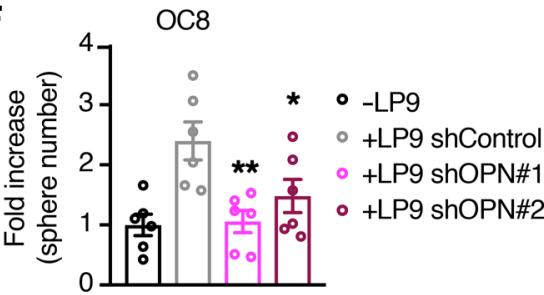

G

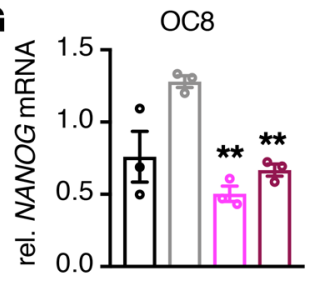

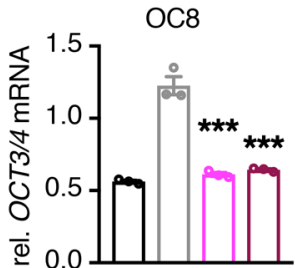
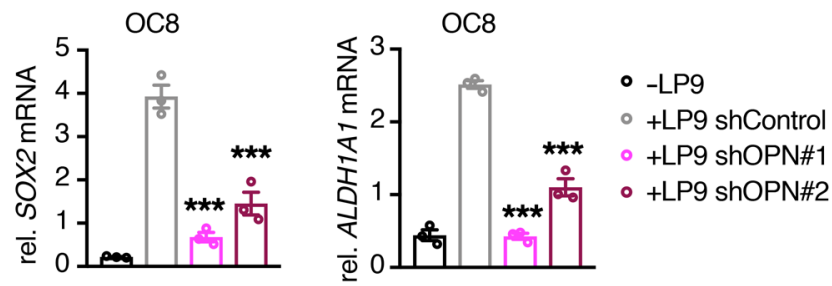

Figure 7. Cancer-associated mesothelial cells promote ovarian cancer cell chemoresistance and stemness through OPN. (A) Effect of exogenous OPN on cisplatin sensitivity of OC8 cells. Cell viability is normalized to its untreated control and statistically compared with the group without OPN treatment ( $n=$ 3-5). (B and C) Sphere-formation assay of OC8 after OPN exposure. Representative sphere images and quantification of sphere-number fold increase are shown $(n=10)$. Scale bars: $200 \mu \mathrm{m}$. (D) Real-time PCR analysis showing relative mRNA expression of stemness markers NANOG, OCT3/4, SOX2, and ALDH1A1 in OC8 cells after OPN exposure, as normalized to GAPDH mRNA $(n=3)$. (E) Cell viability of OC8 cells treated with cisplatin after coculture with LP9 control knockdown (shControl) or LP9 shOPN. Cell viability is normalized to its untreated control and statistically compared with the LP9 shControl group $(n=6)$. (F) Sphere-formation assay of OC8 after coculture with LP9 shControl or LP9 shOPN. Quantification of sphere-number fold increase is shown ( $n=$ 6). Each group is statistically compared with the LP9 shControl group. (C) Real-time PCR analysis showing relative mRNA expression of stemness markers NANOG, OCT3/4, SOX2, and ALDH1A1 in OC8 with LP9 shControl or LP9 shOPN coculture, as normalized to GAPDH mRNA and statistically compared with the LP9 shControl group $(n=3)$. Data are presented as mean \pm SEM. ${ }^{*} P<0.05 ;{ }^{* *} P<0.01 ;{ }^{* *} P<0.001$, 2-way ANOVA (A and E), 2-tailed Student's $t$ test (B and $\mathbf{C}$ ), and 1-way ANOVA (F and $\mathbf{G})$.

Cancer-associated mesothelial cells secrete OPN in the ovarian cancer microenvironment. To identify cancer-associated mesothelial cell-derived factor or factors that promote ovarian cancer chemoresistance, we first fractionated LP9-conditioned media with 3, 10, and $100 \mathrm{kDa}$ cutoff filters to determine the effect of each fraction on OC8 cisplatin sensitivity. Only the fractions of more than $3 \mathrm{kDa}$ and more than $10 \mathrm{kDa}$ maintained the chemoprotective effect of LP9-conditioned media on OC8 cells (Supplemental Figure 6A). Furthermore, proteinase $\mathrm{K}$ digestion abrogated the protective effect, suggesting that protein or proteins between 10 and $100 \mathrm{kDa}$ are responsible for LP9-mediated chemoresistance (Supplemental Figure 6A).
To identify proteins that may contribute to cancer-associated mesothelial cell-mediated chemoprotection, we first compared cytokines secreted by HPMC1 and CAM1 mesothelial cells using Ab cytokine arrays. Several cytokines were detected at higher levels in CAM1-conditioned media compared with the HPMC1-conditioned media (Figure 5A), reinforcing the notion of a cytokine-rich microenvironment favored by CAMs (10). We noted that one of the cytokines with the greatest increase in CAM1-conditioned media was OPN (Figure 5A). We confirmed by ELISA that cancer-associated mesothelial cells (CAM1, CAM2, and LP9) secrete higher levels of OPN compared with HPMCs (HPMC1, HPMC2, 
A

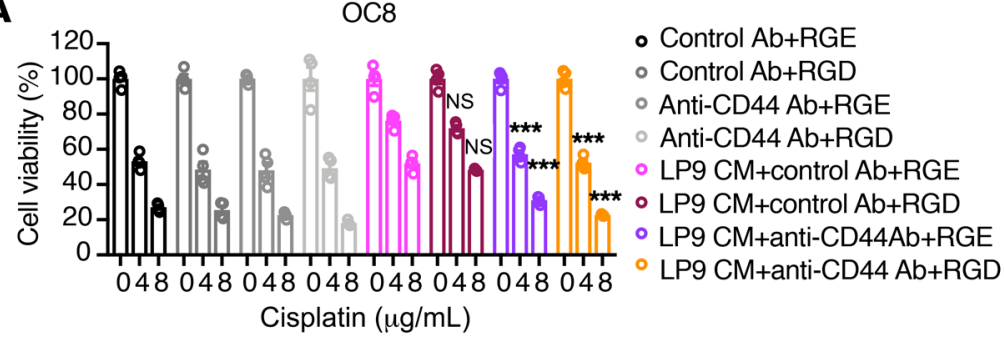

B

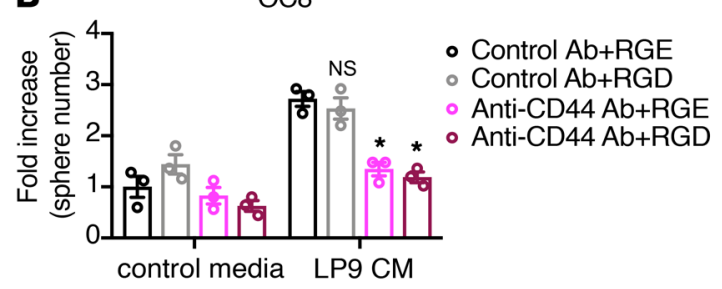

C

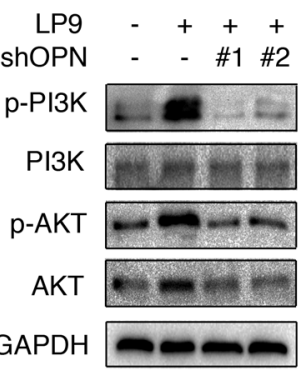

$\mathbf{F}$
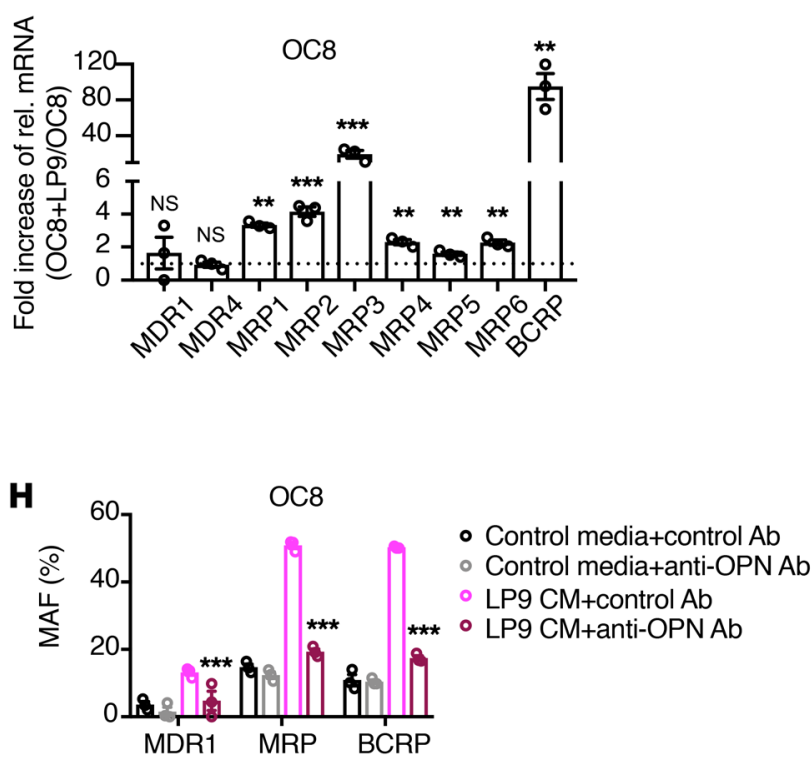

I

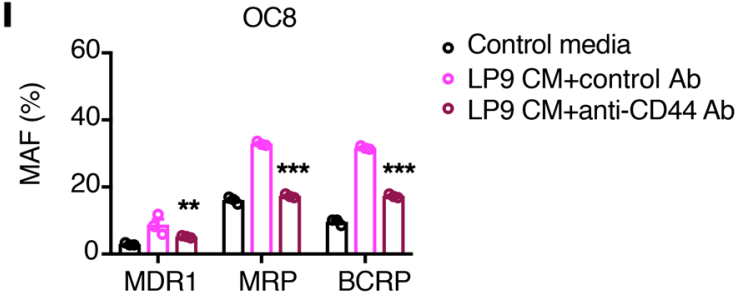

E
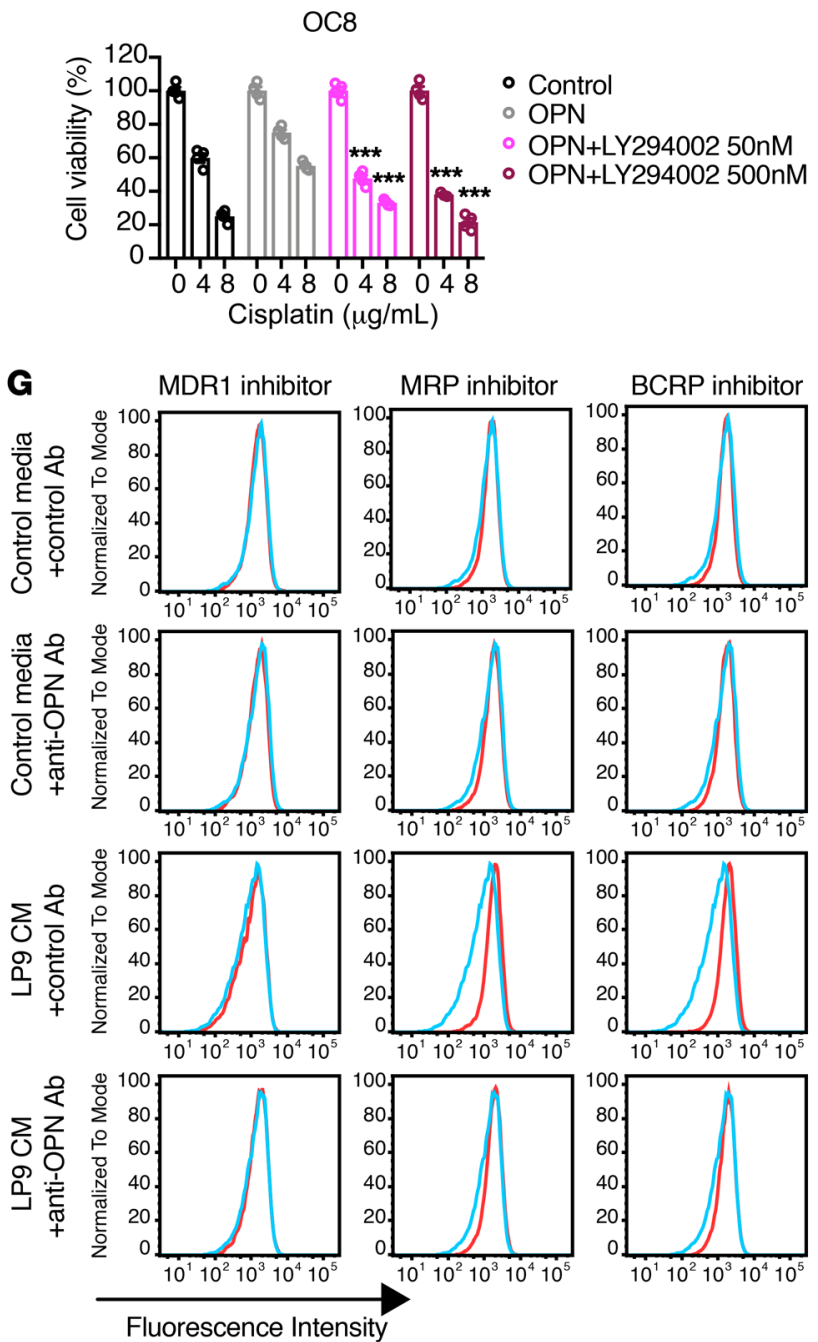

J

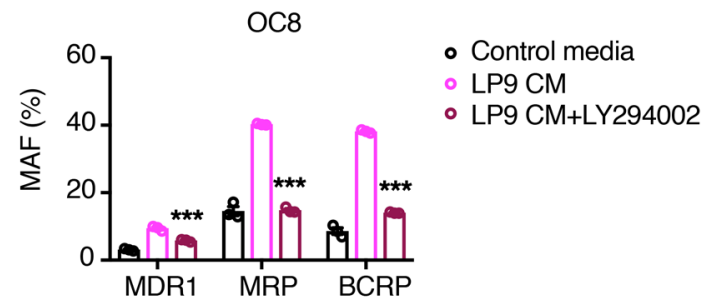


Figure 8. Cancer-associated mesothelial cells promote ovarian cancer cell chemoresistance and $A B C$ drug transporter activity through OPN, CD44, and PI3K/AKT signaling. (A and B) Effect of the anti-CD44 blocking $A b$ and/or integrin-blocking RGD peptide on LP9-conditioned media-mediated cisplatin resistance $(\mathbf{A})$ or sphere-formation increase (B) of OC8 cells. Each group is statistically compared with LP9-conditioned media plus control Ab plus RGE group ( $n=3$ or 4 ). (C) Western blot showing PI3K/AKT signaling in OC8 cells cocultured with LP9 shControl or LP9 shOPN cells. (D) Western blot showing PI3K/AKT signaling in OC8 cells treated with exogenous OPN and/or anti-CD44 blocking Ab. (E) Effect of PI3K/AKT pathway inhibitor LY294002 on OPN-mediated cisplatin resistance of OC8 cells. Each group is statistically compared with exogenous OPN-alone group $(n=4)$. (F) Fold increase of relative mRNA expression of $A B C$ transporters in $O C 8$ with LP9 coculture versus OC8 monoculture, as normalized to GAPDH mRNA in real-time PCR analysis $(n=3)$. ( $(\mathbf{G}$ and $\mathbf{H})$ Multidrug resistance assay detecting the activity of major types of ABC transporters (MDR1, MRP, and BCRP) in OC8 cells treated with LP9-conditioned media and/or antiOPN Ab. Cyan histograms show dye retention of untreated cell, and red histograms show dye retention of respective inhibitor-treated cells in G. The same untreated cells in cyan are used in each row of histograms. Multidrug resistance activity factor (MAF) indicative of corresponding $A B C$ drug transporter activity is shown in $\mathbf{H}$ and statistically compared with LP9-conditioned media plus control Ab group $(n=3)$. (I and J) Multidrug resistance assay detecting ABC transporter activity in OC8 cells treated with LPg-conditioned media and/or anti-CD44 blocking Ab (I) or the PI3K/AKT inhibitor LY294002 (J). MAF indicates corresponding $A B C$ protein activity and is statistically compared with LP9-conditioned media or LP9-conditioned media plus control Ab group $(n=3)$. Data are presented as mean $\pm \mathrm{SEM}$. ${ }^{*} P<0.05$; ${ }^{* *} P<0.01$; ${ }^{* *} P<0.001$, 2-way ANOVA (A, B, E, and $\mathbf{H}-\mathbf{J}$ ) and 2-tailed Student's $t$ test (F).

and HPMC3; Figure 5B). In addition, OPN mRNA expression was increased in cancer-associated mesothelial cells compared with HPMCs (Figure 5, C and D, and ref. 37).

Next, we compared OPN levels between cancer-associated mesothelial cells and ovarian cancer cells. Using cytokine Ab microarrays, we found that OPN was among the top cytokines increased in LP9-conditioned media compared with OVCAR8-conditioned media (Supplemental Figure 6B). We confirmed by ELISA, real-time PCR analysis, and high-resolution single-cell microscopy that cancer-associated mesothelial cells express and secrete higher levels of OPN compared with ovarian cancer cells (Supplemental Figure 6, C-G). Collectively, these findings demonstrate that cancer-associated mesothelial cells express higher levels of OPN compared with HPMCs and ovarian cancer cells.

Ovarian cancer cells disseminate through the malignant ascites to peritoneal tissues, most often to the omentum. To investigate whether mesothelial cells in the omental metastatic microenvironment express OPN, we costained omentum from tumor-free donors and HGSOC patients with the mesothelial marker calretinin and OPN. Calretinin and OPN were coexpressed at the outer layer of omentum near tumor cells, suggesting that mesothelial cells at metastatic sites produce OPN (Supplemental Figure 6, $\mathrm{H}$ and $\mathrm{I}$ ). In contrast, mesothelial cells lining the omentum from tumor-free donors showed lower levels of OPN staining (Supplemental Figure 6, H and I). We further expanded our analysis of OPN expression to the malignant ascites cells from patients with advanced-stage epithelial ovarian cancer (Figure 5E and Supplemental Table 2). Consistent with previous reports $(6,38)$, we found that calretinin-positive mesothelial cells were a substantial portion of total ascites cells (8.56\%-80.81\%; Figure 5E and Supplemental Table 2). The majority of OPN-expressing cells in the ascites fluid were calretinin positive, indicating that mesothelial cells are a major source of OPN in malignant ascites (Figure 5F). We further quantified secreted OPN levels in paired cell-free ascites with total ascites cells and found a strong correlation between calretinin-positive mesothelial cells and OPN concentration within the malignant ascites of individual patients (Figure 5G). Realtime PCR and array-profiling analyses confirmed a positive correlation between $O P N$ and calretinin mRNA expression in ascites cells and peritoneal and omental metastases (Figure 5H, Supplemental Figure 6, J and K, and refs. 39, 40). In addition, high $O P N$ expression in peritoneal metastatic lesions was associated with poor patient survival (Supplemental Figure 6L and ref. 40). Taken together, these data suggest that mesothelial cells are an abundant source of OPN in the ovarian cancer microenvironment.

Ovarian cancer cells induce OPN expression in mesothelial cells through TGF- $\beta$ signaling. Since mesothelial cells in the ovarian cancer microenvironment secrete higher levels of OPN than HPMCs, we hypothesized that ovarian cancer cells induce OPN expression and secretion in mesothelial cells. Indeed, indirect coculture of HPMCs (HPMC1, HPMC2, and HPMC3) with HGSOC cells (OC8, CAOV3, and SNU119) increased OPN expression and secretion in HPMCs (Figure 6, A and B).

TGF- $\beta$ is a potent inducer of OPN expression (41-43) and has been shown to activate mesothelial cells to promote ovarian cancer metastasis $(9,37,44)$. To determine whether ovarian cancer cells induce OPN expression in mesothelial cells via TGF- $\beta$ signaling, we first compared the expression of the TGFB1-3 isoforms in mesothelial cells and ovarian cancer cells by real-time PCR analysis. TGFB1 was the only isoform that was consistently higher in ovarian cancer cells compared with HPMCs (Figure 6C). ELISA confirmed higher levels of secreted TGF- $\beta 1$ by HGSOC cells (OC8, CAOV3, and SNU119) compared with HPMCs (HPMC1, HPMC2, and HPMC3; Figure 6D). Treatment with recombinant human TGF- $\beta 1$ was sufficient to increase OPN secretion by HPMCs (Figure 6E). Moreover, the TGF- $\beta$ receptor inhibitor SB431542 reduced OPN secretion in ovarian cancer cell-conditioned HPMCs (Figure $6 \mathrm{~F})$. These data suggest that ovarian cancer-derived TGF- $\beta 1$ signaling induces OPN expression and secretion by mesothelial cells.

OPN signaling is necessary and sufficient for mesothelial cell-mediated ovarian cancer chemoresistance and stemness. To determine whether OPN is necessary and sufficient to promote cancer-associated mesothelial cell-mediated ovarian cancer chemoresistance and stem-like phenotypes, we treated OC8 and OVCAR8 ovarian cancer cells with recombinant human OPN and assessed their chemosensitivity and stem-like phenotypes. Exogenous OPN enhanced cisplatin resistance of OC8 and OVCAR8 cells (Figure 7A and Supplemental Figure 7A). In addition, OPN treatment increased OC8 and OVCAR8 sphere formation and the expression of the stemness markers, including NANOG, OCT3/4, SOX2, and ALDH1A1 (Figure 7, B-D, and Supplemental Figure 7, B-D). OPN knockdown, which did not impact LP9 cell viability (Supplemental Figure 8, A and B), reduced the ability of LP9 mesothelial cells to promote OC8 and OVCAR8 cell cisplatin resistance (Figure 7E and Supplemental Figure 8, C and D). As a control, the addition of OPN to LP9 
A

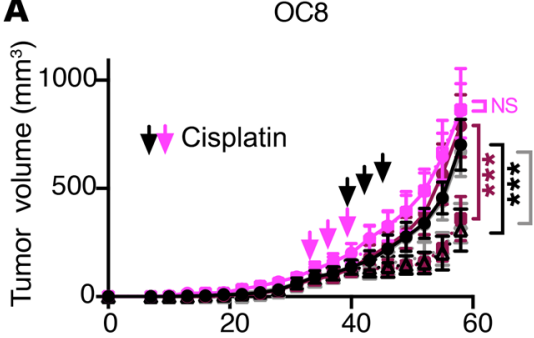

Days after cell inoculation $\left.\begin{array}{l}\text { - LP9 CM+control Ab } \\ \text {-LP9 CM+control Ab cisplatin }\end{array}\right]^{\mathrm{NS}}$

- LP9 CM+anti-OPN Ab

- LP9 CM+anti-OPN Ab cisplatin $]_{*}^{*}$

- Control media+control Ab

$\cdot \Delta \cdot$ Control media+control Ab cisplatin $]_{*}^{*}$

- Control media+anti-OPN Ab

$\triangle$ Control media+anti-OPN Ab cisplatin $]_{*}^{*}$

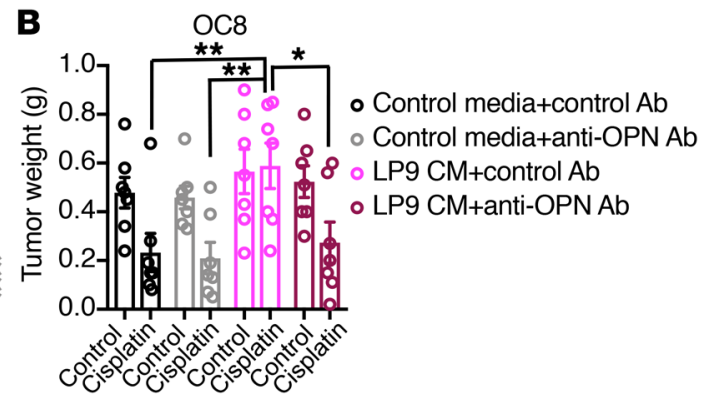

C

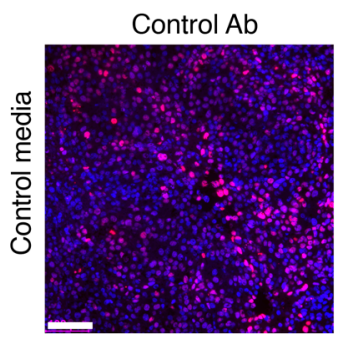

Control Ab
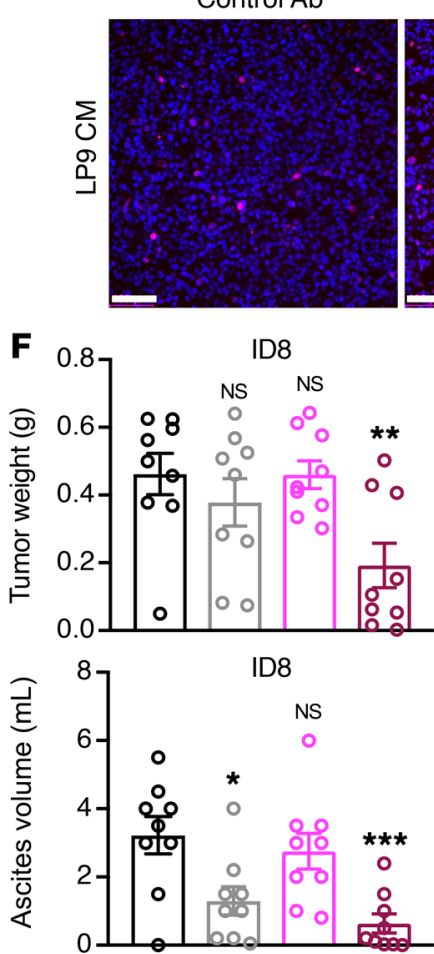

Anti-OPN Ab

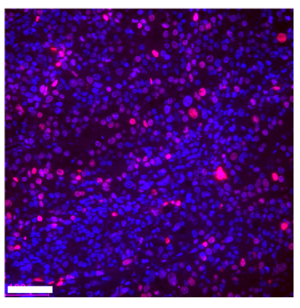

Anti-OPN Ab

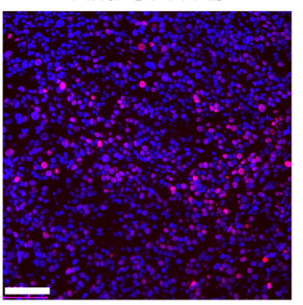

D

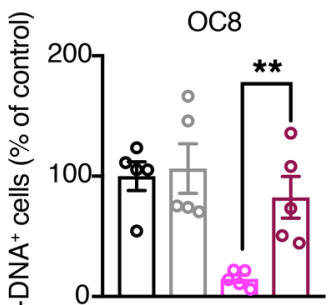

- Control media+control Ab

- Control media+anti-OPN Ab

- LP9 CM+control Ab

- LP9 CM+anti-OPN Ab
E

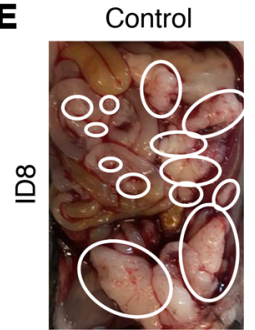

Cisplatin+mut Apt

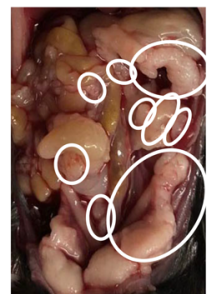

OPN Apt

Cisplatin+OPN Apt
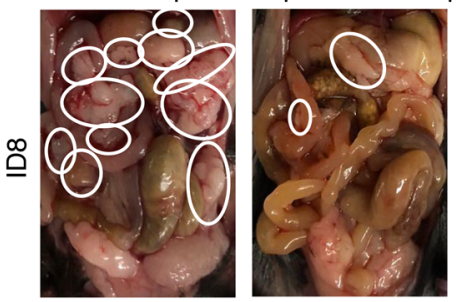

ID8

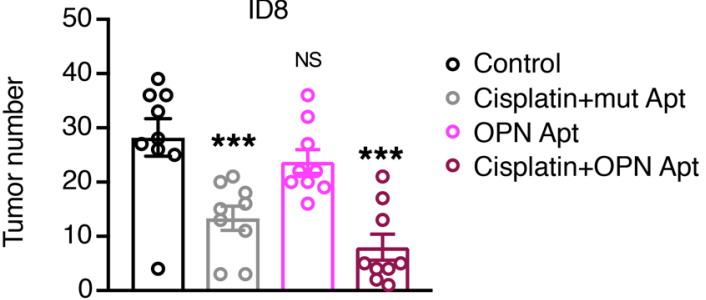

ID8

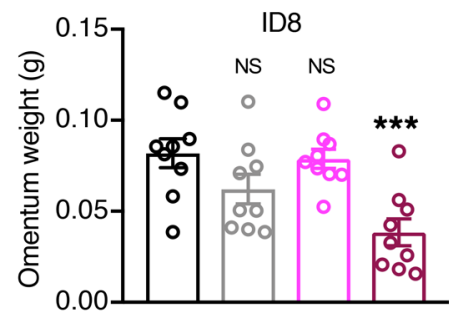

Figure 9. Therapeutic inhibition of OPN enhances the efficacy of cisplatin in human and mouse ovarian cancer xenografts. (A and B) Effect of preincubation with an anti-OPN Ab in the absence or presence of LP9-conditioned media on cisplatin sensitivity of OC 8 subcutaneous tumors in immunodeficient mice ( $n=7$ mice per group). Tumor growth curves are shown in A. Xenograft weights at the end point are shown in B. Arrows show scheme of cisplatin treatment every 3 days for 3 cycles: magenta for LP9-conditioned media with control Ab groups, black for other groups. (C and D) Representative images (C) and quantification of cisplatin-DNA adduct immunofluorescence staining (D) in tumors of OC8 model. Quantification of positive cells (percentages of control media with control Ab group) is based on 5 random fields from 3 tumors in each group. Each dot represents 1 field. Nuclei were stained with DAPI (blue). Scale bars: $100 \mu \mathrm{m}$. (E and F) Representative images of tumor metastases (highlighted by white circles in E) in female [57BL/6] mice injected intraperitoneally with ID8 cells and then treated with a mutant OPN aptamer (mut apt) as control, cisplatin, OPN aptamer (OPN apt), or combination therapy ( $n=9$ mice per group). Tumor weight, tumor number, ascites volume, and omentum weights at the end point are shown in F. Each group is statistically compared with control group in $\mathbf{F}$. Data are presented as mean $\pm \mathrm{SEM}$. ${ }^{*} P<0.05$; ${ }^{* *} P<0.01 ;{ }^{* * *} P$ $<0.001$, 2-way ANOVA (A and B), 2-tailed Student's $t$ test (D) and 1-way ANOVA test (F).

OPN knockdown-derived (shOPN-derived) conditioned media largely restored the chemoprotective effect of LP9-conditioned media on OC8 cells (Supplemental Figure 8C). OPN knockdown also inhibited LP9-mediated tumor-sphere formation and stem- ness marker expression in OC8 and OVCAR8 cells (Figure 7, F and G, and Supplemental Figure 9, A-D). These data suggest that OPN is a key factor driving mesothelial cell-mediated ovarian cancer chemoresistance and stemness. 
OPN signaling increases ovarian cancer chemoresistance through the activation of the CD44 receptor, PI3K-AKT signaling, and ABC drug transporter activity. We hypothesized that the ability of CAM-secreted OPN to promote chemoresistance relies on downstream signals transduced via CD44 and/or integrin receptors on ovarian cancer cells (22). We first examined CD44 and integrin family expression levels on the surface of OC8 and OVCAR8 cells and found that the percentages of OC8 and OVCAR8 cells that were positive for CD44 increased following LP9 coculture (Supplemental Figure 10, A, D, and E). Real-time PCR analysis confirmed an increase in CD44 mRNA expression in LP9-cocultured OC8 and OVCAR8 cells (Supplemental Figure 10F). Several integrin family members, including $\alpha_{\mathrm{v}} \beta_{1}, \alpha_{\mathrm{v}} \beta_{3}, \alpha_{\mathrm{v}} \beta_{5}, \alpha_{5} \beta_{1}, \alpha_{\mathrm{v}} \beta_{6}$, and $\alpha_{8} \beta_{1}$, bind to OPN in an Arg-Gly-Asp (RGD) sequence-dependent manner $(45,46)$. Thus, we evaluated cell-surface expression of integrins $\alpha_{\mathrm{v}}$ and $\beta_{1}$, which are present in all of these RGD-dependent integrin heterodimers. Both integrin $\alpha_{\mathrm{v}}$ and $\beta_{1}$ subunits were expressed by the majority of OC8 and OVCAR8 cells in the absence or presence of LP9 coculture (Supplemental Figure 10, B, C, G, and H).

To determine which receptor or receptors contribute to OPN-mediated chemoresistance, we determined the cisplatin sensitivity of ovarian cancer cells conditioned with LP9 media in combination with a control, anti-CD44 blocking $\mathrm{Ab}$ and/or a competitive inhibitor (RGD peptide) targeting the RGD-dependent integrin receptors. In the OC8, CAOV3, and SNU119 cells, blocking CD44 was sufficient to reverse LP9-mediated cisplatin resistance, while the integrin-inhibiting RGD peptide did not affect LP9-mediated ovarian cancer cell cisplatin resistance (Figure 8A and Supplemental Figure 11, A and B). In the OVCAR8 model, both CD44 and integrin inhibition reduced LP9-mediated chemoresistance (Supplemental Figure 11C). CD44 blockade also repressed ovarian cancer cell cisplatin resistance induced by exogenous OPN (Supplemental Figure $12, \mathrm{~A}-\mathrm{D})$. In addition to reducing cancer-associated mesothelial-mediated chemoresistance, CD44 blockade with the anti-CD44 blocking $\mathrm{Ab}$ reduced LP9-mediated ovarian cancer cell sphere formation (Figure 8B and Supplemental Figure 13, A-C). Together, these findings suggest that CD44 signaling in ovarian cancer cells plays an important role in mediating the chemoprotective effect of cancerassociated mesothelial cells and OPN.

OPN has been reported to induce cancer progression via CD44 receptor and PI3K/AKT signaling $(18,47-50)$. We found that LP9 cocultures activated PI3K/AKT signaling in ovarian cancer cells in an OPN-dependent manner (Figure 8C and Supplemental Figure 14A). In addition, recombinant human OPN induced PI3K/AKT signaling in ovarian cancer cells and CD44 blockade with the antiCD44 blocking Ab reduced PI3K/AKT activation by OPN (Figure $8 \mathrm{D}$ and Supplemental Figure 14B). To determine whether PI3K/AKT signaling contributes to OPN-induced chemoresistance, we determined the cisplatin sensitivity of ovarian cancer cells treated with human recombinant OPN and the PI3K/AKT inhibitor LY294002 (51). LY294002 restored cisplatin sensitivity in OPN-treated ovarian cancer cells (OC8, OVCAR8, CAOV3, and SNU119; Figure 8E and Supplemental Figure 14, C-E). In summary, these data suggest that CD44 and PI3K/AKT signaling play a role in mediating OPN-induced chemoresistance of ovarian cancer cells.

It is well established that enhanced drug efflux mediated by the ATP-binding cassette (ABC) transporters contributes to ovarian cancer cell chemoresistance (52). OPN has been reported to upregulate $\mathrm{ABC}$ transporter expression and drug efflux in prostate cancer cells (53). Thus, we hypothesized that cancer-associated mesothelial cell-secreted OPN promotes ovarian cancer chemoresistance by inducing $\mathrm{ABC}$ transporter activity in ovarian cancer cells. We first examined $A B C$ transporter gene expression in ovarian cancer cells cocultured with cancer-associated mesothelial cells. Several ABC family transporters that are associated with ovarian cancer poor prognosis, including multi-drug resistance protein 1 (MRP1, ABCC1; ref. 54), MRP4 (ABCC4; ref. 55), MRP6 (ABCC6), and breast cancer resistance protein (BCRP, ABCG2; refs. 56, 57), were induced in OC 8 and OVCAR8 cells cocultured with LP9 mesothelial cells (Figure $8 \mathrm{~F}$ and Supplemental Figure $14 \mathrm{~F})$. In flow cytometry-based multidrug resistance assay analysis, LP9-conditioned media decreased the retention of the EFLUXXID fluorescent dye (58) in OC8 and OVCAR8 cells in an OPN-dependent manner, indicating that cancer-associated mesothelial cells promote ovarian cancer cell drug efflux through OPN signaling (Figure 8, G and H, and Supplemental Figure 14G). Moreover, we found that the enhanced drug efflux in LP9-conditioned ovarian cancer cells was dependent upon $\mathrm{ABC}$ drug transporter activity, suggesting that enhanced $\mathrm{ABC}$ transporter activity contributes to cancer-associated mesothelial cell and OPN-mediated ovarian cancer cell chemoresistance (Figure 8, G and H, and Supplemental Figure 14G). Treatment with either the CD44-blocking Ab or the PI3K/AKT inhibitor LY294002 rescued the multidrug-resistant phenotype induced by LP9-conditioned media (Figure 8, I and J, and Supplemental Figure 14, H and I). Together, these findings suggest a model in which cancer-associated mesothelial cells secrete OPN and enhance platinum drug efflux through the activation of CD44/PI3K-AKT/ABC drug transporter activity.

Therapeutic targeting of OPN improves the efficacy of cisplatin in human and mouse ovarian tumor xenografts. To determine the therapeutic potential of the anti-OPN neutralizing $\mathrm{Ab}$ to restore cisplatin sensitivity in mesothelial cell-conditioned ovarian cancer cells, we compared the cisplatin sensitivity of ovarian cancer cells cultured with cancer-associated mesothelial cell-conditioned media alone or together with an anti-OPN neutralizing $\mathrm{Ab}$. The anti-OPN Ab increased the cancer-associated mesothelial cell-primed ovarian cancer cell sensitivity to cisplatin in vitro (Supplemental Figure 15, A and B). Moreover, the anti-OPN Ab reduced the tumor-formation rate, enhanced the efficacy of cisplatin, and increased cisplatin-modified DNA in OC8 xenografts preconditioned with LP9-conditioned media (Figure 9, A-D, and Supplemental Figure 15C). This observation is consistent with our in vitro studies showing $\mathrm{OPN}$ regulation of $\mathrm{ABC}$ drug transporters in ovarian cancer cells (Figure $8 \mathrm{H}$ ).

To determine whether OPN blockade could enhance the efficacy of cisplatin treatment in a more physiologically relevant model, we utilized the ID8 syngeneic murine model of ovarian cancer peritoneal metastasis. The ID8 ovarian cancer model is widely utilized as a model of ovarian cancer, as it metastasizes throughout the peritoneal cavity, significantly affects the bowel, and forms ascites similar to that seen in HGSOC patients (59). However, it should be noted that, at the molecular level, the ID8 model does not harbor mutations commonly associated with HGSOC, including Trp53, Brca1, Brca2, Nf1, or Rb1 (60). Similarly to what occurs with ascites of ovarian cancer patients, the ascites fluid in the ID8 
murine model of ovarian cancer contained OPN-positive mesothelial cells (Supplemental Figure 15D). ID8 tumor-bearing mice were treated with an aptamer against OPN alone or in combination with cisplatin (61-64). A mutant aptamer with poor OPN-binding ability was used as control for the OPN-targeted aptamer in this model (61-64). The combined treatment of the OPN-targeted aptamer with cisplatin decreased total tumor weight and the tumor-bearing omental weight and also reduced tumor number and ascites volume compared with cisplatin with the mutant aptamer or the OPN-targeted aptamer alone (Figure 9, E and F). In summary, our observations suggest that targeting OPN signaling may be an effective therapeutic strategy for potentiating the efficacy of cisplatin in the treatment of metastatic ovarian cancer.

\section{Discussion}

Accumulating evidence suggests that the tumor microenvironment plays an important role in shaping tumor heterogeneity and drug resistance (65). Thus, identifying and targeting factors in the tumor microenvironment that promote resistance to platinum-based chemotherapy has the potential to improve outcomes in patients with advanced ovarian cancer. Peritoneal mesothelial cells line the visceral and parietal surfaces of the peritoneal cavity and are also a major cellular component of malignant ascites fluid $(66,67)$. In our study, we found that $8.56 \%$ to $80.81 \%$ of ovarian cancer patient ascites cells were calretinin-positive mesothelial cells. Here, we demonstrate that patient-derived mesothelial cells from ascites fluid secrete OPN to enhance ovarian cancer cell stemness and platinum resistance. Importantly, therapeutic blockade of OPN enhanced ovarian cancer cell platinum sensitivity in coculture models and in tumor xenografts. These findings identify mesothelial cells as a key cellular component of the ovarian tumor microenvironment that promote chemoresistance and suggest that targeting OPN may be an effective therapeutic strategy for restoring platinum sensitivity in ovarian cancer.

We found that cancer-associated mesothelial cells from HGSOC patients expressed higher levels of OPN compared with mesothelial cells from patients with benign disease. Moreover, ovarian cancer cells induced OPN expression and secretion in mesothelial cells in a TGF- $\beta 1$-dependent manner. TGF- $\beta 1$ is highly expressed in ovarian cancer malignant ascites and omental metastases $(68,69)$. Recent studies have shown that TGF- $\beta 1$ stimulation increases fibronectin expression and mesenchymal transition of mesothelial cells to facilitate ovarian cancer metastasis $(9,44)$. Our data extend these findings by showing that TGF- $\beta 1$ production by ovarian cancer cells increases OPN expression and secretion by mesothelial cells to enhance ovarian cancer chemoresistance.

Our study identifies OPN as an important paracrine-signaling molecule by which mesothelial cells in the ovarian tumor microenvironment promote cancer chemoresistance. OPN has a well-established role in cancer stemness and chemoresistance. In colon cancer, glioma, lung cancer, and gastric cancer, OPN secreted from tumor-associated cells enhances cancer cell stemness in a CD44-dependent manner (47, 70-72). In mesothelio$\mathrm{ma}$, gastric cancer, lung cancer, and prostate cancer, OPN promotes cancer chemoresistance through enhanced hyaluronate (HA)/CD44/PI3K signaling, induction of Bcl-xL, modulation of the $\mathrm{BCL}-2 / \mathrm{BAX}$ ratio, or upregulation of $\mathrm{ABC}$ transporter expression $(53,72-74)$. In ovarian cancer, OPN expression correlates with poor patient prognosis and therapeutic failure (15), but its cellular source and molecular mechanism are unclear. Here, we define a mechanism by which mesothelial cell-derived OPN paracrine signaling promotes ovarian cancer chemoresistance through the activation of the CD44 receptor on the surface of ovarian cancer cells, PI3K/AKT signaling, and downstream $\mathrm{ABC}$ drug efflux transporter activity.

Accumulating evidence supports a role for ovarian CSCs in intraperitoneal metastasis, chemoresistance, and tumor recurrence $(75,76)$. Based on their intrinsic stem-like properties, CSCs are thought to initiate tumor formation, metastasis, and multidrug resistance (77). In HGSOCs, CSCs are enriched within ascites fluid and express intracellular detoxifying scavengers, such as aldehyde dehydrogenase 1 family member A1 (ALDH1A1), canonical stem cell markers, including NANOG, OCT3/4, and SOX2, and drug efflux transporters, such as ABCG2 $(32,33,78$, 79). CSC populations exist in a plastic state and are dynamically shaped by signal cues from the tumor microenvironment $(77,80)$. In HGSOCs, recent studies have highlighted a role for stromal cells within the peritoneal cavity, including fibroblasts $(36,81)$, mesenchymal stem cells (82), adipocytes (83), macrophages (81, 84), and myeloid-derived suppressor cells (35), in CSC expansion and maintenance. Here, we found that mesothelial cells secrete OPN to enrich for ovarian cancer cells with stem cell properties. Notably, we observed that mesothelial-derived OPN induced the expression and activity of the functional ovarian CSC marker ALDH1A1. Recent studies have shown that IL-6/STAT3, DNA methyltransferase 1 (DNMT1), BRD4, and $\beta$-catenin signaling promote ALDH1A1 expression in ovarian cancer cells $(36,79,85)$. Future studies are needed to explore how mesothelial cells in the ovarian cancer microenvironment promote ALDH1A1 expression in ovarian cancer cells.

Finally, our findings indicate that therapeutic targeting of OPN may be an effective strategy for enhancing the efficacy of cisplatin for the treatment of metastatic ovarian cancer. We demonstrate that therapeutic inhibition of mesothelial-secreted OPN with OPN-neutralizing Abs is sufficient to reduce tumor formation and enhance the efficacy of cisplatin treatment in human ovarian cancer xenografts. Anti-OPN treatment was associated with increased cisplatin-modified DNA in the tumor xenografts, consistent with our in vitro studies showing reduced $A B C$ drug efflux transporter activity in ovarian cancer cells treated with the antiOPN neutralizing $\mathrm{Ab}$. These findings suggest a model in which therapeutic targeting of mesothelial cell-derived OPN enhances cisplatin response by increasing the drug concentration and DNA damage inside ovarian cancer cells. In addition, we show that administration of an OPN-targeted aptamer increased the efficacy of cisplatin in a syngeneic murine model of ovarian cancer peritoneal metastasis. Indeed, OPN blockade through the use of neutralizing Abs or aptamers has also been shown to be efficacious in mouse models of metastatic breast cancer and glioblastoma (17, $61,86)$, implicating OPN as an actionable drug target. Boumans et al. recently demonstrated the safety of a humanized OPN-neutralizing $\mathrm{Ab}$ in phase I and multiple-dose phase IIA clinical trials for the treatment of rheumatoid arthritis (87). Whether OPN-neutral- 
izing Abs may enhance the efficacy of chemotherapy in the clinical management of ovarian cancer and other abdominal cancers with peritoneal metastases warrants further investigation $(88,89)$.

\section{Methods}

Statistics. Statistical analyses were conducted using Prism 8 software (GraphPad). Two-tailed Student's $t$ test was used for comparison between 2 groups, and 1-way or 2-way ANOVA was used for comparing more than 2 groups. For all experiments, mean values for a minimum of 3 independent replicates are presented and error bars represent the SEM, unless otherwise indicated in the figure legends. $P$ values of less than 0.05 were considered to be statistically significant.

Study approval. Human specimens were obtained from patients under IRB approval in accordance with recognized ethical guidelines per the US Common Rule. Patients were treated at Stanford University or Washington University, and written, informed consent was obtained for tissue banking in accordance with recognized ethical guidelines per the US Common Rule. For experiments in mouse models, all procedures for mice were approved by the Institutional Animal Care and Use Committee of Stanford University in accordance with institutional and NIH guidelines.

For additional materials and methods, please refer to Supplemental Methods. For information on chemicals, drugs, and reagents and antibodies used in the study, see Supplemental Tables 3 and 4.

\section{Author contributions}

$\mathrm{EBR}, \mathrm{OD}, \mathrm{SCH}$, and $\mathrm{AE}$ supervised the study. JQ performed the in vivo and in vitro experiments in $2 \mathrm{D}$ and analyzed the data. BLL and $\mathrm{KMH}$ performed the 3D organoid culture and staining and analyzed the data. CM performed bioinformatics analysis. SN performed the cytokine array of LP9- and OVCAR8-conditioned media. JTE assisted with the acquisition and interpretation of flow cytometry data. YX contributed to breeding of the mouse colony. KCF collected patient specimens. KCF, VK, and OD collected patient specimens and isolated primary mesothelial cells. AE performed the single-cell imaging and quantification. JQ and EBR wrote and revised the paper. All other authors read the manuscript and provided comments.

\section{Acknowledgments}

We thank Weiping Zou from the University of Michigan for sharing the primary HGSOC cell line OC8 developed in his lab. We thank Patrik Johansson for preparing the 3D image of the ovarian cancer organoid. We thank Rie von Eyben for advice with statistical analysis. We also thank the Stanford Human Pathology/Histology Service Center and PAN Facility Services for organoid processing and RNA quality testing. We are thankful to members of the Rankin and Giaccia labs for thoughtful discussions. This work was supported by the Office of the Assistant Secretary of Defense for Health Affairs through the Department of Defense Ovarian Cancer Research Program under award no. W81XWH-15-1-0097, the Mary Kay Ash Charitable Foundation, and the Stanford BioX Interdisciplinary Initiatives Seed Grants Program (to EBR). OD is supported by the Mary Lake Polan Endowment for Gynecologic Cancer Research.

Address correspondence to: Erinn B. Rankin, Stanford School of Medicine, 269 Campus Drive, Room 1245A, Stanford, California 94305, USA. Phone: 650.497.8742; Email: erankin@stanford.edu.
1. Henley SJ, et al. Annual report to the nation on the status of cancer, part II: Progress toward Healthy People 2020 objectives for 4 common cancers. Cancer. 2020;126(10):2020-2266.

2. Bowtell DD, et al. Rethinking ovarian cancer II: reducing mortality from high-grade serous ovarian cancer. Nat Rev Cancer. 2015;15(11):668-679.

3. Matulonis UA, et al. Ovarian cancer. Nat Rev Dis Primers. 2016;2:16061.

4. Morgan RJ Jr, et al. Epithelial ovarian cancer. J Natl Compr Canc Netw. 2011;9(1):82-113.

5. Davidowitz RA, et al. Mesenchymal gene program-expressing ovarian cancer spheroids exhibit enhanced mesothelial clearance. JClin Invest. 2014;124(6):2611-2625.

6. Peterson VM, et al. Ascites analysis by a microfluidic chip allows tumor-cell profiling. Proc Natl Acad Sci U S A. 2013;110(51):E4978-E4986.

7. Iwanicki MP, et al. Ovarian cancer spheroids use myosin-generated force to clear the mesothelium. Cancer Discov. 2011;1(2):144-157.

8. Natarajan S, et al. Collagen remodeling in the hypoxic tumor-mesothelial niche promotes ovarian cancer metastasis. Cancer Res. 2019;79(9):2271-2284.

9. Kenny HA, et al. Mesothelial cells promote early ovarian cancer metastasis through fibronectin secretion. J Clin Invest. 2014;124(10):4614-4628.

10. Mikula-Pietrasik J, et al. Peritoneal mesothelium promotes the progression of ovarian cancer cells in vitro and in a mice xenograft model in vivo.
Cancer Lett. 2014;355(2):310-315.

11. Kim JH, et al. Osteopontin as a potential diagnostic biomarker for ovarian cancer. JAMA. 2002;287(13):1671-1679.

12. Nakae M, et al. Preoperative plasma osteopontin level as a biomarker complementary to carbohydrate antigen 125 in predicting ovarian cancer. $J$ Obstet Gynaecol Res. 2006;32(3):309-314.

13. Hu ZD, et al. Diagnostic value of osteopontin in ovarian cancer: a meta-analysis and systematic review. PLoS One. 2015;10(5):e0126444.

14. Bao LH, et al. Osteopontin in metastatic lesions as a prognostic marker in ovarian cancers. $J$ Biomed Sci. 2007;14(3):373-381.

15. Cerne K, et al. Potential of osteopontin in the management of epithelial ovarian cancer. Radiol Oncol. 2019;53(1):105-115.

16. Schorge JO, et al. Osteopontin as an adjunct to CA125 in detecting recurrent ovarian cancer. Clin Cancer Res. 2004;10(10):3474-3478.

17. Wei J, et al. Osteopontin mediates glioblastoma-associated macrophage infiltration and is a potential therapeutic target. J Clin Invest. 2019;129(1):137-149.

18. Lin YH, Yang-Yen HF. The osteopontin-CD44 survival signal involves activation of the phosphatidylinositol 3-kinase/Akt signaling pathway. J Biol Chem. 2001;276(49):46024-46030.

19. Robertson BW, et al. Regulation of Erk1/2 activation by osteopontin in PC3 human prostate cancer cells. Mol Cancer. 2010;9:260.
20. Choi SI, et al. Osteopontin production by TM4SF4 signaling drives a positive feedback autocrine loop with the STAT3 pathway to maintain cancer stem cell-like properties in lung cancer cells. Oncotarget. 2017;8(60):101284-101297.

21. Moorman HR, et al. Osteopontin: a key regulator of tumor progression and immunomodulation. Cancers (Basel). 2020;12(11):3379.

22. Zhao H, et al. The role of osteopontin in the progression of solid organ tumour. Cell Death Dis. 2018;9(3):356.

23. Chhieng DC, et al. Calretinin staining pattern aids in the differentiation of mesothelioma from adenocarcinoma in serous effusions. Cancer. 2000;90(3):194-200.

24. Barberis MC, et al. Calretinin. A selective marker of normal and neoplastic mesothelial cells in serous effusions. Acta Cytol.1997;41(6):1757-1761.

25. Connell ND, Rheinwald JG. Regulation of the cytoskeleton in mesothelial cells: reversible loss of keratin and increase in vimentin during rapid growth in culture. Cell. 1983;34(1):245-253.

26. Wu YJ, et al. The mesothelial keratins: a new family of cytoskeletal proteins identified in cultured mesothelial cells and nonkeratinizing epithelia. Cell. 1982;31(3 pt 2):693-703.

27. Wang W, et al. Effector T cells abrogate stroma-mediated chemoresistance in ovarian cancer. Cell. 2016;165(5):1092-1105.

28. Hallas-Potts A, et al. Ovarian cancer cell lines derived from non-serous carcinomas migrate 
and invade more aggressively than those derived from high-grade serous carcinomas. Sci Rep. 2019;9(1):5515.

29. Domcke S, et al. Evaluating cell lines as tumour models by comparison of genomic profiles. Nat Commun. 2013;4:2126.

30. Haley J, et al. Functional characterization of a panel of high-grade serous ovarian cancer cell lines as representative experimental models of the disease. Oncotarget. 2016;7(22):32810-32820.

31. Mitra AK, et al. In vivo tumor growth of highgrade serous ovarian cancer cell lines. Gynecol Oncol. 2015;138(2):372-377.

32. Zhang S, et al. Identification and characterization of ovarian cancer-initiating cells from primary human tumors. Cancer Res. 2008;68(11):4311-4320.

33. Kryczek I, et al. Expression of aldehyde dehydrogenase and CD133 defines ovarian cancer stem cells. Int J Cancer. 2012;130(1):29-39.

34. Chefetz I, et al. A Pan-ALDH1A inhibitor induces necroptosis in ovarian cancer stem-like cells. Cell Rep. 2019;26(11):3061-3075.

35. Cui TX, et al. Myeloid-derived suppressor cells enhance stemness of cancer cells by inducing microRNA101 and suppressing the corepressor CtBP2. Immunity. 2013;39(3):611-621.

36. Wang Y, et al. IL-6 mediates platinum-induced enrichment of ovarian cancer stem cells. JCI Insight. 2018;3(23):e122360.

37. Rynne-Vidal A, et al. Mesothelial-to-mesenchymal transition as a possible therapeutic target in peritoneal metastasis of ovarian cancer. J Pathol. 2017;242(2):140-151.

38. Sheid B. Angiogenic effects of macrophages isolated from ascitic fluid aspirated from women with advanced ovarian cancer. Cancer Lett. 1992;62(2):153-158.

39. Brodsky AS, et al. Expression profiling of primary and metastatic ovarian tumors reveals differences indicative of aggressive disease. PLoS One. 2014;9(4):e94476.

40. Tothill RW, et al. Novel molecular subtypes of serous and endometrioid ovarian cancer linked to clinical outcome. Clin Cancer Res. 2008;14(16):5198-5208.

41. Lenga $Y$, et al. Osteopontin expression is required for myofibroblast differentiation. Circ Res. 2008;102(3):319-327.

42. Neumann C, et al. Osteopontin is induced by TGF- $\beta 2$ and regulates metabolic cell activity in cultured human optic nerve head astrocytes. PLoS One. 2014;9(4):e92762.

43. Shi X, et al. Hoxa-9 represses transforming growth factor-beta-induced osteopontin gene transcription. J Biol Chem. 2001;276(1):850-855.

44. Sandoval P, et al. Carcinoma-associated fibroblasts derive from mesothelial cells via mesothelial-to-mesenchymal transition in peritoneal metastasis. J Pathol. 2013;231(4):517-531.

45. Kazanecki CC, et al. Control of osteopontin signaling and function by post-translational phosphorylation and protein folding. J Cell Biochem. 2007;102(4):912-924.

46. Wei R, et al. Osteopontin -- a promising biomarker for cancer therapy. J Cancer. 2017;8(12):2173-2183.

47. Pietras A, et al. Osteopontin-CD44 signaling in the glioma perivascular niche enhances cancer stem cell phenotypes and promotes aggressive tumor growth. Cell Stem Cell. 2014;14(3):357-369.

48. Ahmed M, et al. An osteopontin/CD44 axis in RhoGDI2-mediated metastasis suppression. Cancer Cell. 2016;30(3):432-443.

49. Pio GM, et al. Soluble bone-derived osteopontin promotes migration and stem-like behavior of breast cancer cells. PLoS One. 2017;12(5):e0177640.

50. Herishanu Y, et al. Activation of $\mathrm{CD} 44$, a receptor for extracellular matrix components, protects chronic lymphocytic leukemia cells from spontaneous and drug induced apoptosis through MCL1. Leuk Lymphoma. 2011;52(9):1758-1769.

51. YuX, et al. Osteopontin promotes hepatocellular carcinoma progression via the PI3K/AKT/Twist signaling pathway. Oncol Lett. 2018;16(4):5299-5308.

52. Ween MP, et al. The role of ABC transporters in ovarian cancer progression and chemoresistance. Crit Rev Oncol Hematol. 2015;96(2):220-256.

53. Hsieh IS, et al. Upregulation of drug transporter expression by osteopontin in prostate cancer cells. Mol Pharmacol. 2013;83(5):968-977.

54. Faggad A, et al. Expression of multidrug resistance-associated protein 1 in invasive ovarian carcinoma: implication for prognosis. Histopathology. 2009;54(6):657-666.

55. Bagnoli M, et al. Clinicopathological impact of ABCC1/MRP1 and ABCC4/MRP4 in epithelial ovarian carcinoma. Biomed Res Int. 2013;2013:143202.

56. Chung WM, et al. Increase paclitaxel sensitivity to better suppress serous epithelial ovarian cancer via ablating androgen receptor/Aryl hydrocarbon receptor-ABCG2 axis. Cancers (Basel). 2019;11(4):E463.

57. Li X, et al. NOS1 upregulates ABCG2 expression contributing to DDP chemoresistance in ovarian cancer cells. Oncol Lett. 2019;17(2):1595-1602.

58. Zhou W, et al. NEK2 induces drug resistance mainly through activation of efflux drug pumps and is associated with poor prognosis in myeloma and other cancers. Cancer Cell. 2013;23(1):48-62.

59. Roby KF, et al. Development of a syngeneic mouse model for events related to ovarian cancer. Carcinogenesis. 2000;21(4):585-591.

60. Walton J, et al. CRISPR/Cas9-mediated Trp53 and Brca2 knockout to generate improved murine models of ovarian high-grade serous carcinoma. Cancer Res. 2016;76(20):6118-6129.

61. Mi Z, et al. RNA aptamer blockade of osteopontin inhibits growth and metastasis of MDA-MB231 breast cancer cells. Mol Ther. 2009;17(1):153-61.

62. Syn WK, et al. Osteopontin is induced by hedgehog pathway activation and promotes fibrosis progression in nonalcoholic steatohepatitis. Hepatology. 2011;53(1):106-115.

63. Li J, et al. Osteopontin RNA aptamer can prevent and reverse pressure overload-induced heart failure. Cardiovasc Res. 2017;113(6):633-643.

64. Coombes JD, et al. Osteopontin neutralisation abrogates the liver progenitor cell response and fibrogenesis in mice. Gut. 2015;64(7):1120-1131.

65. Metcalf KJ, et al. Leveraging microenvironmental synthetic lethalities to treat cancer. J Clin Invest. 2021;131(6):e143765.

66. Chau YY, et al. Visceral and subcutaneous fat have different origins and evidence supports a mesothelial source. Nat Cell Biol. 2014;16(4):367-375.
67. Gupta OT, Gupta RK. Visceral adipose tissue mesothelial cells: living on the edge or just taking up space? Trends Endocrinol Metab. 2015;26(10):515-523.

68. Yamamura $S$, et al. The activated transforming growth factor-beta signaling pathway in peritoneal metastases is a potential therapeutic target in ovarian cancer. Int J Cancer. 2012;130(1):20-28.

69. Abendstein B, et al. Regulation of transforming growth factor-beta secretion by human peritoneal mesothelial and ovarian carcinoma cells. Cytokine. 2000;12(7):1115-1119.

70. Todaro M, et al. CD44v6 is a marker of constitutive and reprogrammed cancer stem cells driving colon cancer metastasis. Cell Stem Cell. 2014;14(3):342-356.

71. Hu J, et al. A CD $44 \mathrm{v}^{+}$subpopulation of breast cancer stem-like cells with enhanced lung metastasis capacity. Cell Death Dis. 2017;8(3):e2679.

72. Park JW, et al. Multi-omics analysis identifies pathways and genes involved in diffuse-type gastric carcinogenesis induced by E-cadherin, p53, and Smad4 loss in mice. Mol Carcinog. 2018;57(7):947-954.

73. Tajima K, et al. Osteopontin-mediated enhanced hyaluronan binding induces multidrug resistance in mesothelioma cells. Oncogene. 2010;29(13):1941-1951.

74. Gu T, et al. Osteopontin is involved in the development of acquired chemo-resistance of cisplatin in small cell lung cancer. Lung Cancer. 2009;66(2):176-183.

75. Varas-Godoy M, et al. The crosstalk between ovarian cancer stem cell niche and the tumor microenvironment. Stem Cells Int. 2017;2017:5263974.

76. Lupia M, Cavallaro U. Ovarian cancer stem cells: still an elusive entity? Mol Cancer. 2017;16(1):64

77. Qian J, Rankin EB. Hypoxia-induced phenotypes that mediate tumor heterogeneity. Adv Exp Med Biol. 2019;1136:43-55.

78. Landen CN Jr, et al. Targeting aldehyde dehydrogenase cancer stem cells in ovarian cancer. Mol Cancer Ther. 2010;9(12):3186-3199.

79. Yokoyama $Y$, et al. BET inhibitors suppress ALDH activity by targeting ALDH1A1 super-enhancer in ovarian cancer. Cancer Res. 2016;76(21):6320-6330.

80. Klymenko Y, Nephew KP. Epigenetic crosstalk between the tumor microenvironment and ovarian cancer cells: a therapeutic road less traveled. Cancers (Basel). 2018;10(9):295.

81. Mazzoldi EL, et al. A juxtacrine/paracrine loop between C-Kit and stem cell factor promotes cancer stem cell survival in epithelial ovarian cancer. Cell Death Dis. 2019;10(6):412.

82. McLean K, et al. Human ovarian carcinomaassociated mesenchymal stem cells regulate cancer stem cells and tumorigenesis via altered BMP production. JClin Invest. 2011;121(8):3206-3219.

83. Cardenas $\mathrm{C}$, et al. Adipocyte microenvironment promotes $\mathrm{Bcl}_{\mathrm{xl}}$ expression and confers chemoresistance in ovarian cancer cells. Apoptosis. 2017;22(4):558-569.

84. Raghavan S, et al. Ovarian cancer stem cells and macrophages reciprocally interact through the WNT pathway to promote pro-tumoral and malignant phenotypes in 3D engineered 
microenvironments. JImmunother Cancer. 2019;7(1):190.

85. Condello $S$, et al. $\beta$-Catenin-regulated ALDH1A1 is a target in ovarian cancer spheroids. Oncogene. 2015;34(18):2297-2308.

86. Dai J, et al. A humanized anti-osteopontin antibody inhibits breast cancer growth and metastasis in vivo. Cancer Immunol Immunother. 2010;59(3):355-366.

87. Boumans MJ, et al. Safety, tolerability, pharmacokinetics, pharmacodynamics and efficacy of the monoclonal antibody ASK8007 blocking osteopontin in patients with rheumatoid arthritis: a randomised, placebo controlled, proof-of-con- cept study. Ann Rheum Dis. 2012;71(2):180-185.

88. Montori G, et al. The treatment of peritoneal carcinomatosis in advanced gastric cancer: state of the art. Int J Surg Oncol. 2014;2014:912418.

89. Koppe MJ, et al. Peritoneal carcinomatosis of colorectal origin: incidence and current treatment strategies. Ann Surg. 2006;243(2):212-222. 